\title{
Adoption rates of electronic health records in Turkish Hospitals and the relation with hospital sizes
}

Ilker Kose ${ }^{1^{*}} \mathbb{D}$, John Rayner ${ }^{2}$, Suayip Birinci ${ }^{3}$, Mustafa Mahir Ulgu ${ }^{3}$, Ismayil Yilmaz ${ }^{3}$, Seyma Guner ${ }^{4}$, HIMSS Analytics $\mathrm{Team}^{4}$ and $\mathrm{MoH}$ Team ${ }^{3}$

\begin{abstract}
Background: Nation-wide adoption of electronic health records (EHRs) in hospitals has become a Turkish policy priority in recognition of their benefits in maintaining the overall quality of clinical care. The electronic medical record maturity model (EMRAM) is a widely used survey tool developed by the Healthcare Information and Management Systems Society (HIMSS) to measure the rate of adoption of EHR functions in a hospital or a secondary care setting. Turkey completed many standardizations and infrastructural improvement initiatives in the health information technology (IT) domain during the first phase of the Health Transformation Program between 2003 and 2017. Like the United States of America (USA), the Turkish Ministry of Health (MoH) applied a bottom-up approach to adopting EHRs in state hospitals. This study aims to measure adoption rates and levels of EHR use in state hospitals in Turkey and investigate any relationship between adoption and use and hospital size.

Methods: EMRAM surveys were completed by 600 (68.9\%) state hospitals in Turkey between 2014 and 2017. The availability and prevalence of medical information systems and EHR functions and their use were measured. The association between hospital size and the availability/prevalence of EHR functions was also calculated.

Results: We found that $63.1 \%$ of all hospitals in Turkey have at least basic EHR functions, and 36\% have comprehensive EHR functions, which compares favourably to the results of Korean hospitals in 2017, but unfavorably to the results of US hospitals in 2015 and 2017. Our findings suggest that smaller hospitals are better at adopting certain EHR functions than larger hospitals.

Conclusion: Measuring the overall adoption rates of EHR functions is an emerging approach and a beneficial tool for the strategic management of countries. This study is the first one covering all state hospitals in a country using EMRAM. The bottom-up approach to adopting EHR in state hospitals that was successful in the USA has also been found to be successful in Turkey. The results are used by the Turkish $\mathrm{MoH}$ to disseminate the nation-wide benefits of EHR functions.
\end{abstract}

Keywords: Electronic health records; meaningful use; CPOE; PACS, eMAR, CDSS, EMRAM, HIMSS

\footnotetext{
* Correspondence: ikose@medipol.edu.tr

'Department of Health System Engineering, Istanbul Medipol University, 34810 Istanbul, Turkey

Full list of author information is available at the end of the article
}

(c) The Author(s). 2020 Open Access This article is licensed under a Creative Commons Attribution 4.0 International License, which permits use, sharing, adaptation, distribution and reproduction in any medium or format, as long as you give appropriate credit to the original author(s) and the source, provide a link to the Creative Commons licence, and indicate if changes were made. The images or other third party material in this article are included in the article's Creative Commons licence, unless indicated otherwise in a credit line to the material. If material is not included in the article's Creative Commons licence and your intended use is not permitted by statutory regulation or exceeds the permitted use, you will need to obtain permission directly from the copyright holder. To view a copy of this licence, visit http://creativecommons.org/licenses/by/4.0/. The Creative Commons Public Domain Dedication waiver (http://creativecommons.org/publicdomain/zero/1.0/) applies to the data made available in this article, unless otherwise stated in a credit line to the data. 


\section{Background}

Electronic health records (EHRs), as defined by the Turkish Ministry of Health $(\mathrm{MoH})$, is any information recorded, stored, transmitted, accessed, correlated, and processed by using electronic systems related to past, present and future physical and mental health condition or diseases of individuals [1]. This information repository, when used in a meaningful manner, keeps all of the records that are useful, effective, ethical, and easily accessible within regulated boundaries [2, 3]. The key functions contained in EHRs are computerized physician order entries (CPOE) [4-7], closed-loop medication administration records (CLMA) [8-12], clinical decision support systems (CDSS) [13-15], picture archiving and communication systems (PACS) [16], and electronic medication administration records (eMAR) [9, 17, 18]. Hospital information systems (HIS) combine these functions with additional modules essential to clinical and administrative processes. Common modules include patient administration systems (PAS) capable of recording the identification and demographic data of patients [19]. Despite varying content and structure due to the local health insurance system, electronic medical billing (EMB) systems are another crucial HIS component integrated with EHRs and PAS [20].

\section{Overall electronic health record capabilities of countries}

There are only a few studies evaluating the national adoption level of EHR functions in hospitals. One of the first and widely cited studies was conducted in 2009 by Jha et al., which surveyed the availability of 24 EHR functions in US hospitals [21]. Results were classified according to whether the hospitals had basic or comprehensive EHR functions. Basic EHR functions indicate that clinical documentation, CPOE, CDSS, and laboratory and imaging results are limited to one clinic, while comprehensive EHR functions indicate availability in all clinics of the hospital. The study showed that only $1.5 \%$ of US hospitals had comprehensive EHR functions, and 7.6\% had basic EHR functions. A subsequent study in 2011, which was also conducted by tha et al., indicated that the total percentage of US hospitals having at least basic EHR functions had increased to 15.1\% [22]. AdlerMilstein et al. conducted more recent studies in 2014 [23], 2015 [24], and 2017 [25]. These studies showed that the proportion of US hospitals having comprehensive EHR functions was $25.5 \%$ in $2014,34.1 \%$ in 2015 , and $39.1 \%$ in 2017 . Similarly, the proportion of hospitals having at least basic EHR functions was $58.9 \%$ in 2014, $75.2 \%$ in 2015 , and $80.5 \%$ in 2017.

Studies focusing on Korean hospitals are also noteworthy. The first study was conducted by Park et al. in 2005 [26]. Researchers used a survey designed by Ash et al. [27], which focused more on CPOE than other
EHR functions. This study showed that although $80.3 \%$ of hospitals have CPOE forms, only 9\% have complete EHR systems. Two subsequent studies conducted in Korean hospitals used the Jha et al. [21] survey, which allowed them to compare their results to the Jha et al. US hospital results. A 2012 study by Yoon et al. [28] showed that the percentage of Korean hospitals having at least basic EHR functions was 37.2\%, which was higher than the proportion of hospitals in the USA (15.1\%). The most recent study published in 2017 by Kim et al. [29] showed that the percentage of Korean hospitals having at least basic EHR functions had increased to $58.1 \%$. Still, this figure was lower than the proportion of US hospitals with at least basic EHR functions $(80.5 \%)$ for the same year. The rapid increase in the adoption rate of EHRs in US hospitals may be attributed to the financial and political support provided by the HITECH Act (2009).

Another notable study was published in 2014 by Shu et al. [30]. This cross-sectional study measured the rate of EHR adoption in tertiary hospitals in China. The authors conducted a national survey entitled the Model of EHR Grading (MEG), which gives hospitals a rating between Stage 0 and 7 based on their adoption of 37 EHR functions. This study showed that, among 848 hospitals, $30.7 \%$ were Stage 0, $12.0 \%$ were Stage 1, 31.7\% were Stage 2, 22.2\% were Stage 3, 2.7\% were Stage 4, 0.6\% were Stage $5,0.1 \%$ were Stage 6 , and $0 \%$ were Stage 7 . Other studies performed in Greece [31] and Saudi Arabia [32] were not survey-based, but rather thematic reviews focused on providing insights for policymakers.

Kanakubo and Kharrazi write one of the most recent studies comparing country-wide EHR adoption level in 2019. This study compares two countries by gathering data set from the Healthcare Information and Management Systems Society (HIMSS) for the USA, and from the Ministry of Health, Labour and Welfare (MHLW) for Japan for the years of 2008, 2011, and 2014. The results of this study showed that while the USA and Japan have similar status in 2008 and 2011, the USA became better than Japan in adopting EHR for small, medium, and large hospitals in 2014. On the other hand, the government hospitals in Japan are better than US hospitals for all 3 years [33].

Approaches to encouraging the adoption of EHRs in hospitals is a critical point for policymakers. United Kingdom (UK) and US strategies to stimulate the adoption of EHR functions are of particular interest. Aziz et al., in 2014, asserted that many lessons should be learned from US achievements with a bottom-up approach and criticized the UK's top-down decisionmaking [34]. They attributed the US success to the three distinct stages of the implementation-strategy and stated that the UK strategy lacked clearly defined milestones 
Owen et al. responded to this criticism of the UK approach by highlighting the $100 \%$ adoption rate of EHRs at the primary care level when US rates remained considerably lower [35]. In a recent study published in 2018, Wilson and Khansa also compared the EHR systems of the UK and the USA. They noted that the top-down strategy brought early success to the UK with general practitioners, but noted that this strategy was not successful when the UK tried to bring EHRs to hospitals because of the complexity of processes among stakeholders. They suggested that even though the USA had the most extensive private healthcare system in the world, which might be more challenging to control, their bottom-up approach seemed more successful than the top-down approach of the UK, which had the most extensive public healthcare system in the world [36].

\section{Electronic health record capability in Turkey}

The $\mathrm{MoH}$ in Turkey launched the Health Transformation Program in 2003 [37] and finalized the first phase in 2017. Many milestones were achieved with this national healthcare reform program that related to the use of information and technology standards such as the National Health Data Dictionary (NHDD) [38], the Health Coding Reference Server [39], the International Classification of Diseases, 10th Revision (ICD-10) [40], Health Level Seven International (HL7) [41, 42], National Health Tariffs [43], and other systems including the Family Physician Information System [44], the National Health Information System [38], the Central Physician Booking System [45], the Central Claims Management System [46], a teleradiology system [47], and a Personal Health Records system [48, 49]. As an essential part of the national health information technology infrastructure, the $\mathrm{MoH}$ initiated a pilot national e-prescription system in June 2012, which became mandatory as of January 2013. Although the target is set as $95 \%$, the adoption rate of the e-prescription system was $87 \%$ as of September 2018 [50].

Although there are many nation-wide standards and applications in Turkey, there had been no measurement of adoption rates for new systems within healthcare facilities (i.e., hospitals, health centers, etc.). The $\mathrm{MoH}$ of Turkey decided to conduct a study measuring the overall adoption rates of EHR functions within state hospitals. One of the objectives of the MoH Strategic Plan for 2013-2017 was to study the "digital hospital" concept and disseminate it across all state hospitals [51]. In line with this strategic objective, the $\mathrm{MoH}$ signed a collaborative agreement with the Healthcare Information and Management Systems Society (HIMSS) in 2013 [52] and decided on using EMRAM. EMRAM is a powerful survey which is applied by more than 25 countries including some EU countries, Turkey, China, Russia, Saudi
Arabia, etc., and very common in the USA so that there are 2039 and 285 validated hospitals against the requirements of Stage 6 and Stage 7 correspondingly in the USA only [53]. Besides Turkey, Portugal is also considering to use EMRAM as a country-wide measure for digital transformation [54], and Canada applied a customized version of it for community-based physicians [55]. Since it is an EHR adoption model, EMRAM does not focus on human and organizational capabilities but the technological functionality of the hospital [56].

The Turkish MoH's role as a policymaker allowed hospitals to conduct relevant studies within the period determined by the Strategic Plan. As such, Turkey also applied a US-style bottom-up approach to encouraging the adoption of EHRs in state hospitals.

\section{Electronic medical health record adaptation model}

The electronic medical health record adaptation model (EMRAM) [57] developed by HIMSS provides algorithms to assess inpatient services in acute hospitals based on their EHR capabilities and, like all other models [58-60] created by HIMSS, has eight stages from 0 to 7. EMRAM is first developed in 2005 and enhanced by HIMSS to meet the technological progress of the overall digitalization of hospitals [61]. The assessment criteria for hospitals in our study are shown in Table 1 [57]. The EMRAM survey is currently used in over 50 countries worldwide, has been used over 60,000 times to assess digital maturity and nearly 3000 times to validate hospitals at either Stage 6 or Stage 7. HIMSS suspects that approximately $830 \mathrm{~m}$ people have been impacted by healthcare providers using one or more maturity models.

The EMRAM is a simple, well-evaluated model that requires users to assess levels of compliance with a straight forward "yes/no" or "Present/Absent" responses. If the response is positive, which means the relevant application or function is available, occasionally, the subject is asked to set the percentage of compliance or usage coverage (i.e., departmental, hospital-wide, etc.).

\section{Methods}

This study utilizes the EMRAM survey, which includes five dimensions: Software Applications (SA), Software Usage (SU), Electronic Ordering (EO), Image Management Systems (IMS), and Medication Safety (MS). While the SA dimension investigates the availability of software and EHR functions, the SU dimension focused on the prevalence of them. The EO dimension focuses on eorder usage in drug and non-drug orders, and CDSS usage in e-orders. The IMS dimension investigates the archiving and retrieving the clinical images. Finally, the MS focuses on CLMA records through the hospital. 
Table 1 HIMSS EMRAM Requirements (as of January 1, 2018)

\begin{tabular}{ll}
\hline Stage & Cumulative Capabilities \\
\hline Stage 7 & $\begin{array}{l}\text { Complete Electronic Medical Record (EMR); Continuity of Care Document transactions to share data; Data } \\
\text { warehousing; Data Continuity with Emergency Department, Ambulatory, and OP }\end{array}$ \\
$\begin{array}{l}\text { Stage } 6 \\
\text { (Comprehensive EHR functions) }\end{array}$ & $\begin{array}{l}\text { Physician documentation (structured templates); Full Clinical Decision Support System (CDSS) (variance \& } \\
\text { Compliance); Full Remote-PACS }\end{array}$ \\
Stage 5 & $\begin{array}{l}\text { Closed-Loop Medication Administration (CLMA) } \\
\text { Stage } 4\end{array}$ \\
Stage 3 \\
(basic EHR functions) \\
Stage 2
\end{tabular}

To compare this study with previous studies conducted with US and Korean hospitals, EMRAM requirements are aligned with the 24 functions of the survey developed by Jha et al. [21] so that Stage 3, 4 and 5 of EMRAM indicates that the hospital has basic EHR functions, and Stage 6 indicates that the hospital has comprehensive EHR functions. Kanakubo and Kharrazi do a similar alignment in 2019 to benchmark between the USA and Japan [33].

The survey studies took place between 2014 and 2017. Sixteen regional workshops were organized in the same period, with 2716 participants, including hospital managers, from 870 distinct state hospitals. This study aimed to cover all 870 state hospitals [62] in Turkey, and all hospitals are invited to fulfill the survey. Surveys that were of inadequate quality were excluded from this study. In cases where the same hospital submitted more than one survey having the same score, only the most recent survey was analyzed.

We preferred using the "adoption of EHR" phrase instead of "use of EHR" or "adoption and use of EHR" in our study for two reasons: 1) The EMRAM is the abbreviation for the electronic medical record "adoption" model. The adoption, here, means the availability and use of EHR functions within the hospital. 2) While some respectful authors preferred using "Use of EHR" [21, 63, 64] in the meaning of "the availability and use of some EHR functions" in their papers, some other authors $[24,25,28,65,66]$ used "EHR adoption" in the same meaning as we preferred.

IBM SPSS version 23 was used to perform statistical analyses. The Chi-Square test was conducted to analyze associations or differences between the categorical variables, such as hospital size and the adoption rate of EHR functions. The threshold for significance was set at $\alpha=0.05$.

\section{Results}

A total of 889 surveys were collected from 870 hospitals. Of the 889 surveys collected, 204 were excluded as duplicate submissions. The number of distinct hospitals that completed surveys was 685 (78.7\%). Of these, 85 surveys were excluded because of poor quality data and severe inconsistencies. Overall, 600 verified and acceptable surveys $(68.9 \%)$ were analyzed in this study. The distribution of the hospitals and their size, type, and level of healthcare are shown in Table 2. All seven of Turkey's regions, $97.5 \%$ of Turkey's 79 provinces, and $68.9 \%$ of all Turkish hospitals were represented in the sample. Small hospitals represent $49.2 \%$, medium-sized hospitals represent $34.2 \%$, and Large hospitals represent $16.7 \%$ of the participating hospitals. All hospitals participating in the study are state hospitals $(88.5 \%)$, while 11 , $5 \%$ are training hospitals. Of participating hospitals, $81.5 \%$ were secondary hospitals, $9 \%$ were tertiary hospitals, and $9.5 \%$ were specialized hospitals.

The distribution of the number of hospitals in each EMRAM stage is shown in Fig. 1. It is remarkable that most hospitals $(72.66 \%)$ are either at Stage 2 or 6 . The barrier requirements of Stage 3, such as having PACS, eMAR, and adopting nursing documents, appear to be a threshold, as once hospitals achieve Stage 3, the remaining technologies seem to be adopted relatively easily and adoption levels increase steadily. If we consider these results in terms of the categories developed by Jha et al. in 2009, 36\% of hospitals have Comprehensive EHR functions, and 63\% have Basic EHR functions.

\section{Availability of applications and electronic health record functions}

This section provides results regarding the availability of information systems and EHR functions in hospitals.

\section{Hospital information systems, laboratory information systems, and patient administration systems}

The availability results of HIS, Laboratory Information Systems (LIS), and PAS are listed in Table 3 according to hospital size. The results show that $100 \%$ of hospitals 
Table 2 Basic characteristics of responding hospitals

\begin{tabular}{|c|c|c|c|c|}
\hline Characteristic & Total Number in Turkey & Participating Hospitals & Participating Percentage (\%) & Percentage in Sample (\%) \\
\hline Region & 7 & 7 & 100 & 100 \\
\hline Province & 81 & 79 & 97.5 & 100 \\
\hline Hospital & 870 & 600 & 68.9 & 100 \\
\hline \multicolumn{5}{|l|}{ Size } \\
\hline Small (0-99 beds) & 531 & 295 & 55.5 & 49.2 \\
\hline Medium (100-399 beds) & 232 & 205 & 88.3 & 34.2 \\
\hline Large ( $\geq 400$ beds) & 117 & 100 & 85.4 & 16.7 \\
\hline \multicolumn{5}{|l|}{ Teaching Status } \\
\hline Public Hospital & 775 & 531 & 68.5 & 88.5 \\
\hline Training Hospital & 95 & 69 & 72.6 & 11.5 \\
\hline \multicolumn{5}{|l|}{ Hospital Type ${ }^{a}$} \\
\hline Secondary Hospital & 682 & 489 & 71.2 & 81.5 \\
\hline Tertiary Hospital & 95 & 54 & 56.8 & 9 \\
\hline Branch Hospital & 93 & 57 & 61.2 & 9.5 \\
\hline
\end{tabular}

${ }^{a}$ All hospitals in the sample are public (state) hospitals

have a HIS suite. Similarly, $94 \%$ of hospitals have PAS, and $93 \%$ of hospitals have LIS integrated with their HIS. It is also evident that hospital size does is not positively associated with having HIS, LIS, and PAS. The survey investigates whether the application is live only in the relevant department such as laboratory, etc. or live in the entire hospital. Thus such the tables have two corresponding columns as "Live" and "Live -Hospital-wide".

Clinical documents and computerized Physician order entry Results related to clinical documentation (CDR) are provided in Table 4, according to hospital size. The results show that $98.6 \%$ of hospitals have a CDR, and $79.7 \%$ of hospitals have a hospital-wide CDR. CDR is one of the requirements of EMRAM Stage 2 and Physician and nursing documents, as requirements of EMRAM Stage 3 , have very similar availability percentages across hospital sizes. While $86.2 \%$ of hospitals have physician documents, nursing documents are available in $84.8 \%$ of all hospitals. Those high percentages may be explained by the Turkish national healthcare quality standards (SKS), which have required a clinical document infrastructure since 2009. CPOE, on the other hand, is not functional in $13.8 \%$ of hospitals. The size of the hospital has no

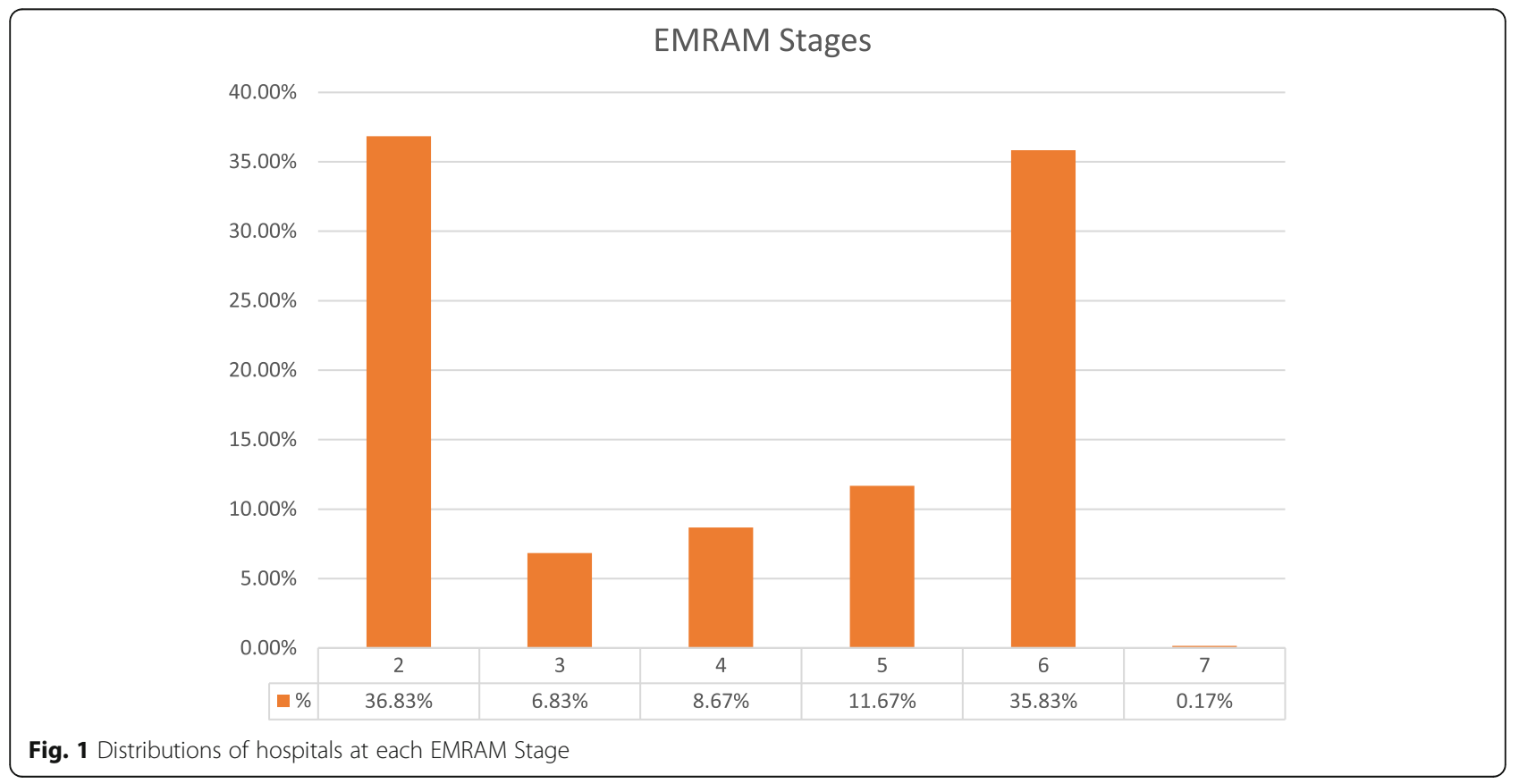


Table 3 Availability of HIS, LIS and PAS

\begin{tabular}{|c|c|c|c|c|c|c|c|}
\hline Applications & Hospital Size & Live & $\begin{array}{l}\text { Live - hospital- } \\
\text { wide }\end{array}$ & $\begin{array}{l}\text { Live - } \\
\text { departmental }\end{array}$ & $\begin{array}{l}\text { Not } \\
\text { Automated }\end{array}$ & Missing & Total \\
\hline \multirow[t]{5}{*}{$\begin{array}{l}\text { EMR / Hospital Information } \\
\text { System (Suite) }\end{array}$} & Large (> = 400 beds) & $\begin{array}{l}100 \\
(100 \%)\end{array}$ & $\begin{array}{l}0 \\
(0.0 \%)\end{array}$ & $\begin{array}{l}0 \\
(0.0 \%)\end{array}$ & $\begin{array}{l}0 \\
(0.0 \%)\end{array}$ & 0 & 100 \\
\hline & $\begin{array}{l}\text { Medium (100-399 } \\
\text { beds) }\end{array}$ & $\begin{array}{l}205 \\
(100 \%)\end{array}$ & $\begin{array}{l}0 \\
(0.0 \%)\end{array}$ & $\begin{array}{l}0 \\
(0.0 \%)\end{array}$ & $\begin{array}{l}0 \\
(0.0 \%)\end{array}$ & 0 & 205 \\
\hline & Small (6-99 beds) & $\begin{array}{l}294 \\
(99.6 \%)\end{array}$ & $\begin{array}{l}1 \\
(0.34 \%)\end{array}$ & $\begin{array}{l}0 \\
(0.0 \%)\end{array}$ & $\begin{array}{l}0 \\
(0.0 \%)\end{array}$ & 0 & 295 \\
\hline & Percentage & $99.8 \%$ & $0.2 \%$ & $0.0 \%$ & $0.0 \%$ & $0.0 \%$ & $100 \%$ \\
\hline & Total & $\begin{array}{l}599 \\
(99.8 \%)\end{array}$ & $\begin{array}{l}1 \\
(0.16 \%)\end{array}$ & $\begin{array}{l}\mathbf{0} \\
(0.0 \%)\end{array}$ & $\begin{array}{l}\mathbf{0} \\
(0.0 \%)\end{array}$ & 0 & 600 \\
\hline \multirow[t]{5}{*}{ Patient Administration System } & Large (> = 400 beds) & $\begin{array}{l}1 \\
(1 \%)\end{array}$ & $\begin{array}{l}98 \\
(98 \%)\end{array}$ & $\begin{array}{l}0 \\
(0.0 \%)\end{array}$ & $\begin{array}{l}1 \\
(1 \%)\end{array}$ & 0 & 100 \\
\hline & $\begin{array}{l}\text { Medium (100-399 } \\
\text { beds) }\end{array}$ & $\begin{array}{l}2 \\
(0.99 \%)\end{array}$ & $\begin{array}{l}189 \\
(93.56 \%)\end{array}$ & $\begin{array}{l}3 \\
(1.48 \%)\end{array}$ & $\begin{array}{l}8 \\
(3.96 \%)\end{array}$ & 3 & 205 \\
\hline & Small (6-99 beds) & $\begin{array}{l}5 \\
(1.70 \%)\end{array}$ & $\begin{array}{l}269 \\
(91.80 \%)\end{array}$ & $\begin{array}{l}9 \\
(3.07 \%)\end{array}$ & $\begin{array}{l}10 \\
(3.41 \%)\end{array}$ & 2 & 295 \\
\hline & Percentage & $1.3 \%$ & $92.7 \%$ & $2.0 \%$ & $3.2 \%$ & $0.8 \%$ & $100 \%$ \\
\hline & Total & $\begin{array}{l}8 \\
(1.34 \%)\end{array}$ & $\begin{array}{l}556 \\
(93.44 \%)\end{array}$ & $\begin{array}{l}12 \\
(2.01 \%)\end{array}$ & $\begin{array}{l}19 \\
(3.19 \%)\end{array}$ & 5 & 600 \\
\hline \multirow[t]{5}{*}{$\begin{array}{l}\text { Laboratory Information } \\
\text { System }\end{array}$} & Large (> = 400 beds) & $\begin{array}{l}41 \\
(41 \%)\end{array}$ & $\begin{array}{l}57 \\
(57 \%)\end{array}$ & $\begin{array}{l}2 \\
(2 \%)\end{array}$ & $\begin{array}{l}0 \\
(0.0 \%)\end{array}$ & 0 & 100 \\
\hline & $\begin{array}{l}\text { Medium (100-399 } \\
\text { beds) }\end{array}$ & $\begin{array}{l}43 \\
(21.07 \%)\end{array}$ & $\begin{array}{l}153 \\
(75 \%)\end{array}$ & $\begin{array}{l}7 \\
(3.43 \%)\end{array}$ & $\begin{array}{l}1 \\
(0.49 \%)\end{array}$ & 1 & 205 \\
\hline & Small (6-99 beds) & $\begin{array}{l}7 \\
(2.37 \%)\end{array}$ & $\begin{array}{l}257 \\
(87.11 \%)\end{array}$ & $\begin{array}{l}29 \\
(9.83 \%)\end{array}$ & $\begin{array}{l}2 \\
(0.67 \%)\end{array}$ & 0 & 295 \\
\hline & Percentage & $15.2 \%$ & $77.8 \%$ & $6.3 \%$ & $0.5 \%$ & $0.2 \%$ & $100 \%$ \\
\hline & Total & $\begin{array}{l}91 \\
(15.19 \%)\end{array}$ & $\begin{array}{l}467 \\
(77.96 \%)\end{array}$ & $\begin{array}{l}38 \\
(6.34 \%)\end{array}$ & $\begin{array}{l}3 \\
(0.5 \%)\end{array}$ & 1 & 600 \\
\hline
\end{tabular}

significant relationship to the availability of clinical documents and CPOE systems.

\section{Medication administration}

Pharmacy and medication administration are essential functions of Hospital Information Systems. As depicted in Table 5, the surveys indicate that $99.5 \%$ of all hospitals have a pharmacy management system, even though three $(0.5 \%)$ of them receive this service from an external vendor. Medications administered to the patient are recorded at the point of service in $66 \%$ of the hospitals, but not in the other $29.2 \%$. The high availability of pharmacy management systems can be explained by $\mathrm{MoH}$ regulations addressing stock management and efficiency criteria for state hospital pharmacies that have been in place since 2013. Despite the high availability of information systems, the lack of medication application recording implies that information systems are still more focused on institutional purposes like billing than on clinical services.

\section{Image management}

As depicted in Table 6, survey results show that $89 \%$ of hospitals have a PACS system; but the PACS systems in
$14.5 \%$ of hospitals are not integrated with the hospital's HIS and may only be available to the radiology department. Only $9.5 \%$ of hospitals have a dictation and speech recognition system to help radiologists write their reports more efficiently, so technology adoption has not yet penetrated the reporting process.

\section{Usage of applications and electronic health record functions}

This section presents results regarding the usage and dissemination of information systems and EHR functions in hospitals.

Clinical documents and computerized Physician order entry As shown in Table 4, Physician Documents (PD) systems are available in $84.6 \%$ of hospitals, but we found significant variations in the levels of PD systems (Table 7). A vast majority of hospitals (79.4\%) have electronic medical record systems, and $74.5 \%$ of hospitals are using PDs in at least $50 \%$ of the hospital. The ratio-generating discrete data obtained from the PDs are also other critical indicators that represent the capacity to extract information from the medical record. We found that 
Table 4 Availability of clinical documents and CPOE systems

\begin{tabular}{|c|c|c|c|c|c|c|c|}
\hline Applications & Hospital Size & Live & $\begin{array}{l}\text { Live - hospital- } \\
\text { wide }\end{array}$ & $\begin{array}{l}\text { Live - } \\
\text { departmental }\end{array}$ & Not Automated & Missing & $\mathrm{p}$ \\
\hline \multirow[t]{5}{*}{$\begin{array}{l}\text { Clinical Data Repository } \\
\text { (CDR) }\end{array}$} & Large (> = 400 beds) & $\begin{array}{l}42 \\
(42 \%)\end{array}$ & $\begin{array}{l}56 \\
(56 \%)\end{array}$ & $\begin{array}{l}1 \\
(1 \%)\end{array}$ & $\begin{array}{l}1 \\
(1 \%)\end{array}$ & 0 & $p<0.001$ \\
\hline & Medium (100-399 beds) & $\begin{array}{l}44 \\
(21.5 \%)\end{array}$ & $\begin{array}{l}154 \\
(75.1 \%)\end{array}$ & $\begin{array}{l}4 \\
(2 \%)\end{array}$ & $\begin{array}{l}3 \\
(1.5 \%)\end{array}$ & 0 & \\
\hline & Small (6-99 beds) & $\begin{array}{l}7 \\
(2.4 \%)\end{array}$ & $\begin{array}{l}268 \\
(91.2 \%)\end{array}$ & $\begin{array}{l}16 \\
(5.4 \%)\end{array}$ & $\begin{array}{l}3 \\
(1 \%)\end{array}$ & 1 & \\
\hline & Percentage & $15.5 \%$ & $79.7 \%$ & $3.5 \%$ & $1.2 \%$ & $0.2 \%$ & \\
\hline & Total & 93 & 478 & 21 & 7 & 1 & \\
\hline \multirow[t]{5}{*}{ Nursing Documentation } & Large (> = 400 beds) & $\begin{array}{l}24 \\
(24 \%)\end{array}$ & $\begin{array}{l}55 \\
(55 \%)\end{array}$ & $\begin{array}{l}2 \\
(2 \%)\end{array}$ & $\begin{array}{l}19 \\
(19 \%)\end{array}$ & 0 & $p<0.001$ \\
\hline & Medium (100-399 beds) & $\begin{array}{l}25 \\
(12.3 \%)\end{array}$ & $\begin{array}{l}131 \\
(64.5 \%)\end{array}$ & $\begin{array}{l}10 \\
(4.9 \%)\end{array}$ & $\begin{array}{l}37 \\
(18.2 \%)\end{array}$ & 2 & \\
\hline & Small (6-99 beds) & $\begin{array}{l}6 \\
(2 \%)\end{array}$ & $\begin{array}{l}230 \\
(78.2 \%)\end{array}$ & $\begin{array}{l}26 \\
(8.8 \%)\end{array}$ & $\begin{array}{l}32 \\
(10.9 \%)\end{array}$ & 1 & \\
\hline & Percentage & $9.2 \%$ & $69.3 \%$ & $6.3 \%$ & $14.7 \%$ & $0.5 \%$ & \\
\hline & Total & 55 & 416 & 38 & 88 & 3 & \\
\hline \multirow[t]{5}{*}{ Physician Documentation } & Large (> = 400 beds) & $\begin{array}{l}28 \\
(28 \%)\end{array}$ & $\begin{array}{l}53 \\
(53 \%)\end{array}$ & $\begin{array}{l}3 \\
(3 \%)\end{array}$ & $\begin{array}{l}16 \\
(16 \%)\end{array}$ & 0 & $p<0.001$ \\
\hline & Medium (100-399 beds) & $\begin{array}{l}32 \\
(15.8 \%)\end{array}$ & $\begin{array}{l}128 \\
(63.1 \%)\end{array}$ & $\begin{array}{l}11 \\
(5.4 \%)\end{array}$ & $\begin{array}{l}32 \\
(15.8 \%)\end{array}$ & 2 & \\
\hline & Small (6-99 beds) & $\begin{array}{l}6 \\
(2.0 \%)\end{array}$ & $\begin{array}{l}234 \\
(79.6 \%)\end{array}$ & $\begin{array}{l}22 \\
(7.5 \%)\end{array}$ & $\begin{array}{l}32 \\
(10.9 \%)\end{array}$ & 1 & \\
\hline & Percentage & $11.0 \%$ & $69.2 \%$ & $6.0 \%$ & $13.3 \%$ & $0.5 \%$ & \\
\hline & Total & 66 & 415 & 36 & 80 & 3 & \\
\hline \multirow[t]{5}{*}{ CPOE } & Large (> = 400 beds) & $\begin{array}{l}25 \\
(25 \%)\end{array}$ & $\begin{array}{l}55 \\
(55 \%)\end{array}$ & $\begin{array}{l}2 \\
(2 \%)\end{array}$ & $\begin{array}{l}18 \\
(18 \%)\end{array}$ & 0 & $p<0.001$ \\
\hline & Medium (100-399 beds) & $\begin{array}{l}31 \\
(15.1 \%)\end{array}$ & $\begin{array}{l}132 \\
(64.4 \%)\end{array}$ & $\begin{array}{l}8 \\
(3.9 \%)\end{array}$ & $\begin{array}{l}34 \\
(16.6 \%)\end{array}$ & 0 & \\
\hline & Small (6-99 beds) & $\begin{array}{l}3 \\
(1 \%)\end{array}$ & $\begin{array}{l}234 \\
(79.6 \%)\end{array}$ & $\begin{array}{l}26 \\
(8.8 \%)\end{array}$ & $\begin{array}{l}31 \\
(10.5 \%)\end{array}$ & 1 & \\
\hline & Percentage & $9.8 \%$ & $70.2 \%$ & $6.0 \%$ & $13.8 \%$ & $0.2 \%$ & \\
\hline & Total & 59 & 421 & 36 & 83 & 1 & \\
\hline
\end{tabular}

75.2\% of the hospitals with PDs are using discrete data in PDs in at least $50 \%$ of the hospital. The regulations of the Turkish $\mathrm{MoH}$ can also explain this high percentage of discrete data usage. The $\mathrm{MoH}$ accredits HIS vendors annually according to their capability to submit relevant datasets to the $\mathrm{MoH}$ as outlined in the NHDD since 2015 [38].

Electronic ordering also has a similar prevalence. The usage of CPOE is $72.5 \%$ for medication and $62.7 \%$ for non-medication orders, respectively. However, orders for nurses in inpatient care facilities have a slightly higher proportion of $79 \%$. Table 9 shows that the percentage of CPOE usage for inpatient medication orders is 66.6 and $70.5 \%$ for non-medication orders in more than $50 \%$ of the hospital.

Although there is no significant relationship between hospital size and the use of electronic ordering (Table 8), the prevalence of electronic ordering has a significant relationship with hospital size (Table 9). The results show that small hospitals are better than medium-sized and larger hospitals in adopting electronic ordering capabilities. Verbal orders are not allowed according to regulations applicable to Turkish state hospitals. However, it seems that managers can enforce this rule better in smaller hospitals.

\section{Clinical decision support}

Table 10 presents the results regarding hospitals' access to CDSS. CDSS was used in $69 \%$ of physician/nursing documents, $71.7 \%$ of medication orders, and $57.3 \%$ of non-medication orders. Additionally, although there is no significant relationship between hospital size and the use of CDSS in clinical documents and non-medication orders, the use of CDSS in medication orders has a significant relationship with hospital size. Small hospitals are better than medium-sized and larger hospitals in adopting CDSS for medication orders. Considering Tables 5, 9, and 10 together, we can infer that despite 
Table 5 Availability of medication administration systems

\begin{tabular}{|c|c|c|c|c|c|c|c|c|c|}
\hline Applications & Hospital Size & Live & $\begin{array}{l}\text { Live -hospital- } \\
\text { wide }\end{array}$ & $\begin{array}{l}\text { Live - } \\
\text { departmental }\end{array}$ & $\begin{array}{l}\text { Installation in } \\
\text { Process }\end{array}$ & $\begin{array}{l}\text { Not } \\
\text { Automated }\end{array}$ & $\begin{array}{l}\text { ESP } \\
\text { attached }\end{array}$ & Missing & Total \\
\hline \multirow[t]{5}{*}{$\begin{array}{l}\text { Pharmacy Management } \\
\text { System }\end{array}$} & $\begin{array}{l}\text { Large (> = 400 } \\
\text { beds) }\end{array}$ & $\begin{array}{l}41 \\
(41 \%)\end{array}$ & $\begin{array}{l}55 \\
(55 \%)\end{array}$ & $\begin{array}{l}4 \\
(4 \%)\end{array}$ & $\begin{array}{l}0 \\
(0 \%)\end{array}$ & $\begin{array}{l}0 \\
(0 \%)\end{array}$ & $\begin{array}{l}0 \\
(0 \%)\end{array}$ & 0 & 100 \\
\hline & $\begin{array}{l}\text { Medium (100-399 } \\
\text { beds) }\end{array}$ & $\begin{array}{l}43 \\
(20.97 \%)\end{array}$ & $\begin{array}{l}144 \\
(70.24 \%)\end{array}$ & $\begin{array}{l}15 \\
(7.3 \%)\end{array}$ & $\begin{array}{l}0 \\
(0 \%)\end{array}$ & $\begin{array}{l}2 \\
(0.97 \%)\end{array}$ & $\begin{array}{l}1 \\
(0.48 \%)\end{array}$ & 0 & 205 \\
\hline & Small (6-99 beds) & $\begin{array}{l}8 \\
(2.71 \%)\end{array}$ & $\begin{array}{l}247 \\
(83.72)\end{array}$ & $\begin{array}{l}37 \\
(12.54 \%)\end{array}$ & $\begin{array}{l}0 \\
(0 \%)\end{array}$ & $\begin{array}{l}1 \\
(0.33 \%)\end{array}$ & $\begin{array}{l}2 \\
(0.67 \%)\end{array}$ & 0 & 295 \\
\hline & Percentage & $15.3 \%$ & $74.3 \%$ & $9.3 \%$ & $0.0 \%$ & $0.5 \%$ & $0.5 \%$ & $0.0 \%$ & $100 \%$ \\
\hline & Total & 92 & 446 & 56 & 0 & 3 & 3 & 0 & 600 \\
\hline \multirow[t]{5}{*}{$\begin{array}{l}\text { Electronic Medication } \\
\text { Administration Record }\end{array}$} & $\begin{array}{l}\text { Large ( }>=400 \\
\text { beds) }\end{array}$ & $\begin{array}{l}30 \\
(30 \%)\end{array}$ & $\begin{array}{l}46 \\
(46 \%)\end{array}$ & $\begin{array}{l}4 \\
(4 \%)\end{array}$ & $\begin{array}{l}1 \\
(1 \%)\end{array}$ & $\begin{array}{l}19 \\
(19 \%)\end{array}$ & $\begin{array}{l}0 \\
(0 \%)\end{array}$ & 0 & 100 \\
\hline & $\begin{array}{l}\text { Medium (100-399 } \\
\text { beds) }\end{array}$ & $\begin{array}{l}29 \\
(14.28 \%)\end{array}$ & $\begin{array}{l}110 \\
(54.18 \%)\end{array}$ & $\begin{array}{l}3 \\
(1.47 \%)\end{array}$ & $\begin{array}{l}0 \\
(0 \%)\end{array}$ & $\begin{array}{l}61 \\
(30.04 \%)\end{array}$ & $\begin{array}{l}0 \\
(0 \%)\end{array}$ & 2 & 205 \\
\hline & Small (6-99 beds) & $\begin{array}{l}5 \\
(1.70 \%)\end{array}$ & $\begin{array}{l}176 \\
(60.06 \%)\end{array}$ & $\begin{array}{l}17 \\
(5.80 \%)\end{array}$ & $\begin{array}{l}0 \\
(0 \%)\end{array}$ & $\begin{array}{l}95 \\
(32.42 \%)\end{array}$ & $\begin{array}{l}0 \\
(0 \%)\end{array}$ & 2 & 295 \\
\hline & Percentage & $10.7 \%$ & $55.3 \%$ & $4.0 \%$ & $0.2 \%$ & $29.2 \%$ & $0.0 \%$ & $0.7 \%$ & $100 \%$ \\
\hline & Total & 64 & 332 & 24 & 1 & 175 & 0 & 4 & 600 \\
\hline
\end{tabular}

the fact that nearly all hospitals have pharmacy management systems and drug databases, small hospitals are adopting e-order and CDSS for medications more quickly than larger hospitals.

\section{Closed-loop medication administration}

Table 11 shows that $69.2 \%$ of hospitals have a second line of validation for prescriptions conducted by pharmacists before the drug is delivered to the wards and patients. On the other hand, only $0.8 \%$ of hospitals have an automatic dispensing system for drugs which means that drugs are delivered from pharmacies to the wards and then from the ward station to the bedside by the staff via trolleys/ carts. This method is acceptable for drug safety, even if it is not very time and cost-efficient when compared to automated medication dispensing (AMD) systems [67, 68].

Furthermore, the survey suggests that only $45 \%$ of hospitals use technology at the bedside when administering medicines (i.e., barcode and Radio-frequency identification (RFID)) to electronically confirm the Institute for Healthcare Improvement's Five Rights of Medication Administration: right patient, right drug, right dose, right time and right path $[69,70]$. Similarly, only $41.3 \%$ of hospitals are immediately recording drug administration at the bedside, which means that nurses are recording the drug administration at the ward station after

Table 6 Availability of image management systems

\begin{tabular}{|c|c|c|c|c|c|c|c|c|c|}
\hline Applications & Hospital Size & Live & $\begin{array}{l}\text { Live - } \\
\text { hospital- } \\
\text { wide }\end{array}$ & $\begin{array}{l}\text { Live - } \\
\text { departmental }\end{array}$ & $\begin{array}{l}\text { Installation } \\
\text { in Process }\end{array}$ & $\begin{array}{l}\text { Service Not } \\
\text { Provided }\end{array}$ & $\begin{array}{l}\text { Not } \\
\text { Automated }\end{array}$ & Missing & Total \\
\hline \multirow{5}{*}{$\begin{array}{l}\text { Dictation with } \\
\text { Speech } \\
\text { Recognition }\end{array}$} & Large ( $>=400$ beds) & $\begin{array}{l}1 \\
(1 \%)\end{array}$ & $\begin{array}{l}6 \\
(6 \%)\end{array}$ & $\begin{array}{l}6 \\
(6 \%)\end{array}$ & $\begin{array}{l}0 \\
(0.0 \%)\end{array}$ & $\begin{array}{l}0 \\
(0.0 \%)\end{array}$ & $\begin{array}{l}46 \\
(46 \%)\end{array}$ & 41 & 100 \\
\hline & Medium (100-399 beds) & $\begin{array}{l}1 \\
(0.64 \%)\end{array}$ & $\begin{array}{l}15 \\
(9.61 \%)\end{array}$ & $\begin{array}{l}5 \\
(3.20 \%)\end{array}$ & $\begin{array}{l}0 \\
(0.0 \%)\end{array}$ & $\begin{array}{l}0 \\
(0.0 \%)\end{array}$ & $\begin{array}{l}135 \\
(86.53 \%)\end{array}$ & 49 & 205 \\
\hline & Small (6-99 beds) & $\begin{array}{l}1 \\
(0.35 \%)\end{array}$ & $\begin{array}{l}16 \\
(5.65 \%)\end{array}$ & $\begin{array}{l}6 \\
(2.12 \%)\end{array}$ & $\begin{array}{l}0 \\
(0.0 \%)\end{array}$ & $\begin{array}{l}0 \\
(0.0 \%)\end{array}$ & $\begin{array}{l}260 \\
(91.87 \%)\end{array}$ & 12 & 295 \\
\hline & Percentage & $0.5 \%$ & $6.2 \%$ & $2.8 \%$ & $0.0 \%$ & $0.0 \%$ & $73.5 \%$ & $17.0 \%$ & $100 \%$ \\
\hline & Total & 3 & 37 & 17 & 0 & 0 & 441 & 102 & 600 \\
\hline \multirow[t]{5}{*}{$\begin{array}{l}\text { Radiology - } \\
\text { Central PACS }\end{array}$} & Large ( $>=400$ beds) & $\begin{array}{l}31 \\
(31 \%)\end{array}$ & $\begin{array}{l}56 \\
(56 \%)\end{array}$ & $\begin{array}{l}1 \\
(1 \%)\end{array}$ & $\begin{array}{l}1 \\
(1 \%)\end{array}$ & $\begin{array}{l}1 \\
(1 \%)\end{array}$ & $\begin{array}{l}9 \\
(9 \%)\end{array}$ & 1 & 100 \\
\hline & Medium (100-399 beds) & $\begin{array}{l}34 \\
(16.58)\end{array}$ & $\begin{array}{l}137 \\
(66.8 \%)\end{array}$ & $\begin{array}{l}24 \\
(11.70 \%)\end{array}$ & $\begin{array}{l}0 \\
(0.0 \%)\end{array}$ & $\begin{array}{l}1 \\
(0.48 \%)\end{array}$ & $\begin{array}{l}9 \\
(4.39 \%)\end{array}$ & 0 & 205 \\
\hline & Small (6-99 beds) & $\begin{array}{l}4 \\
(1.36 \%)\end{array}$ & $\begin{array}{l}185 \\
(63.13 \%)\end{array}$ & $\begin{array}{l}62 \\
(21.16 \%)\end{array}$ & $\begin{array}{l}0 \\
(0.0 \%)\end{array}$ & $\begin{array}{l}1 \\
(0.34 \%)\end{array}$ & $\begin{array}{l}41 \\
(13.99 \%)\end{array}$ & 2 & 295 \\
\hline & Percentage & $11.5 \%$ & $63.0 \%$ & $14.5 \%$ & $0.2 \%$ & $0.5 \%$ & $9.8 \%$ & $0.5 \%$ & $100 \%$ \\
\hline & Total & 69 & 378 & 87 & 1 & 3 & 59 & 3 & 600 \\
\hline
\end{tabular}


Table 7 Prevalence of physician documents

\begin{tabular}{|c|c|c|c|c|c|c|c|}
\hline SW Usage & Hospital Size & $51-100 \%$ & $1-50 \%$ & Not Applicable & Total & Missing & $\mathbf{P}$ \\
\hline \multirow[t]{5}{*}{$\begin{array}{l}\text { What percent of all current medical records } \\
\text { are electronic (incl. Digital/scanned data)? }\end{array}$} & Large ( $>=400$ beds) & $\begin{array}{l}59 \\
(59 \%)\end{array}$ & $\begin{array}{l}0 \\
(0.0 \%)\end{array}$ & $\begin{array}{l}0 \\
(0.0 \%)\end{array}$ & 100 & 41 & $0.017^{*}$ \\
\hline & Medium (100-399 beds) & $\begin{array}{l}152 \\
(74.14 \%)\end{array}$ & $\begin{array}{l}9 \\
(4.39 \%)\end{array}$ & $\begin{array}{l}0 \\
(0.0 \%)\end{array}$ & 205 & 44 & \\
\hline & Small (6-99 beds) & $\begin{array}{l}265 \\
(89.83 \%)\end{array}$ & $\begin{array}{l}29 \\
(9.83 \%)\end{array}$ & $\begin{array}{l}0 \\
(0.0 \%)\end{array}$ & 295 & 1 & \\
\hline & Percentage & $79.3 \%$ & $6.3 \%$ & $0.0 \%$ & $100.0 \%$ & $14.3 \%$ & \\
\hline & Total & 476 & 38 & 0 & 600 & 86 & \\
\hline \multirow[t]{5}{*}{$\begin{array}{l}\text { What percent of Physician Documentation } \\
\text { generates discrete (computer-readable) data? }\end{array}$} & Large (> = 400 beds) & $\begin{array}{l}71 \\
(71 \%)\end{array}$ & $\begin{array}{l}6 \\
(6 \%)\end{array}$ & $\begin{array}{l}16 \\
(16 \%)\end{array}$ & 100 & 7 & 0.321 \\
\hline & Medium (100-399 beds) & $\begin{array}{l}150 \\
(73.17 \%)\end{array}$ & $\begin{array}{l}13 \\
(6.34 \%)\end{array}$ & $\begin{array}{l}34 \\
(16.58 \%)\end{array}$ & 205 & 8 & \\
\hline & Small (6-99 beds) & $\begin{array}{l}230 \\
(77.96 \%)\end{array}$ & $\begin{array}{l}24 \\
(82.75 \%)\end{array}$ & $\begin{array}{l}32 \\
(93.60 \%)\end{array}$ & 295 & 9 & \\
\hline & Percentage & $75.2 \%$ & $7.2 \%$ & $13.7 \%$ & $100.0 \%$ & $4.0 \%$ & \\
\hline & Total & 451 & 43 & 82 & 600 & 24 & \\
\hline \multirow[t]{5}{*}{$\begin{array}{l}\text { What percent of physicians use the Physician } \\
\text { Documentation system? }\end{array}$} & Large (> = 400 beds) & $\begin{array}{l}73 \\
(73 \%)\end{array}$ & $\begin{array}{l}6 \\
(6 \%)\end{array}$ & $\begin{array}{l}16 \\
(16 \%)\end{array}$ & 100 & 5 & 0.826 \\
\hline & Medium (100-399 beds) & $\begin{array}{l}149 \\
(72.68 \%)\end{array}$ & $\begin{array}{l}16 \\
(7.80 \%)\end{array}$ & $\begin{array}{l}34 \\
(16.58 \%)\end{array}$ & 205 & 6 & \\
\hline & Small (6-99 beds) & $\begin{array}{l}225 \\
(92.8 \%)\end{array}$ & $\begin{array}{l}26 \\
(8.81 \%)\end{array}$ & $\begin{array}{l}41 \\
(43.15 \%)\end{array}$ & 295 & 3 & \\
\hline & Percentage & $74.5 \%$ & $8.0 \%$ & $15.2 \%$ & $100.0 \%$ & $2.3 \%$ & \\
\hline & Total & 447 & 48 & 91 & 600 & 14 & \\
\hline
\end{tabular}

${ }^{*} p<0.05$

leaving the patient's side. Interestingly, there is no significant relationship between hospital size and the use of CLMA functions, with the exception of "second line validation for medical prescriptions, which is documented electronically." Table 11 indicates that small hospitals are worse than larger hospitals at providing second line validation of prescriptions electronically. To explain this result, we hypothesize that larger hospitals may have more financial and human resources to implement second-line validation.

Table 12 shows the items or persons (i.e., patient and nurse) to be identified using technologies such as RFID or barcodes during medication administration at bedside. Our results show that technology is used more frequently to identify medications and patients than to identify nurses. While these percentages are higher in large and medium hospitals, they are significantly lower in small hospitals. Moreover, the $p$-value indicates that there is a significant relationship between the auto-identified target (medication, nurse, and patient) and the use of technology. This result implies that nurses do not consider it a necessity to validate themselves and their patients electronically but do validate medication administration at bedside using technology.

\section{Image management}

IMS are stand-alone applications that are integrated with EHRs or HIS for practical usage. Table 13 shows that $74.7 \%$ of hospitals integrated their IMS with EHRs. When we consider the prevalence of IMS in hospitals, we can see that $37.3 \%$ of hospitals are using IMS in greater than $50 \%$ of the entire facility. There is no significant relationship between hospital size and the percentage of IMS integration with EHRs or with the prevalence of IMS. This situation can be explained by a nation-wide teleradiology system implemented by the Turkish MoH since 2008 [47].

\section{Discussion}

This study shows that HIS and main ancillaries, such as laboratory, radiology, and pharmacy information systems, are present in all Turkish hospitals included in this study. The availability of LIS, PAS, PACS, and MIS varies between 63.6 and 94\%. The prevalence of EHR functions such as CPOE, CDSS, clinical documents, and drug management ranges from 70 to $99.5 \%$. The size of the hospital has no significant relationship with the availability of those applications.

On the other hand, results show that not all hospitals that have such applications have adopted them. The 
Table 8 Availability of electronic ordering

\begin{tabular}{|c|c|c|c|c|c|c|}
\hline Questions & Hospital Size & Yes ${ }^{* * *}$ & No*** & Not Applicable*** & Missing ${ }^{* * *}$ & $\mathbf{P}$ \\
\hline \multirow[t]{5}{*}{ Electronic ordering for medication } & Large ( $>=400$ beds) & $\begin{array}{l}54 \\
(90.0 \%)\end{array}$ & $\begin{array}{l}5 \\
(8.3 \%)\end{array}$ & $\begin{array}{l}1 \\
(1.7 \%)\end{array}$ & 40 & $0.004^{* *}$ \\
\hline & Medium (100-399 beds) & $\begin{array}{l}140 \\
(85.9 \%)\end{array}$ & $\begin{array}{l}11 \\
(6.7 \%)\end{array}$ & $\begin{array}{l}12 \\
(7.4 \%)\end{array}$ & 42 & \\
\hline & Small (6-99 beds) & 241 & 41 & 5 & 8 & \\
\hline & Percentage & $72.5 \%$ & $9.5 \%$ & $3.0 \%$ & $15.0 \%$ & \\
\hline & Total & 435 & 57 & 18 & 90 & \\
\hline \multirow[t]{5}{*}{ Electronic ordering for non-medication } & Large (> = 400 beds) & $\begin{array}{l}41 \\
(97.6 \%)\end{array}$ & $\begin{array}{l}1 \\
(2.4 \%)\end{array}$ & $\begin{array}{l}0 \\
(0.0 \%)\end{array}$ & 58 & $0.016^{*}$ \\
\hline & Medium (100-399 beds) & $\begin{array}{l}90 \\
(98.9 \%)\end{array}$ & $\begin{array}{l}1 \\
(1.1 \%)\end{array}$ & $\begin{array}{l}0 \\
(0.0 \%)\end{array}$ & 114 & \\
\hline & Small (6-99 beds) & $\begin{array}{l}245 \\
(91.4 \%)\end{array}$ & $\begin{array}{l}23 \\
(8.6 \%)\end{array}$ & $\begin{array}{l}0 \\
(0.0 \%)\end{array}$ & 27 & \\
\hline & Percentage & $62.7 \%$ & $4.2 \%$ & $0.0 \%$ & $33.2 \%$ & \\
\hline & Total & 376 & 25 & 0 & 199 & \\
\hline \multirow[t]{5}{*}{$\begin{array}{l}\text { Electronic ordering for nursing and/or } \\
\text { physician services }\end{array}$} & Large (> = 400 beds) & $\begin{array}{l}75 \\
(76.5 \%)\end{array}$ & $\begin{array}{l}5 \\
(5.1 \%)\end{array}$ & $\begin{array}{l}18 \\
(18.4 \%)\end{array}$ & 2 & $0.001^{* *}$ \\
\hline & Medium (100-399 beds) & $\begin{array}{l}160 \\
(78.4 \%)\end{array}$ & $\begin{array}{l}17 \\
(8.3 \%)\end{array}$ & $\begin{array}{l}27 \\
(13.2 \%)\end{array}$ & 1 & \\
\hline & Small (6-99 beds) & $\begin{array}{l}239 \\
(81.3 \%)\end{array}$ & $\begin{array}{l}37 \\
(12.6 \%)\end{array}$ & $\begin{array}{l}18 \\
(6.1 \%)\end{array}$ & 1 & \\
\hline & Percentage & $79.0 \%$ & $9.8 \%$ & $10.5 \%$ & $0.7 \%$ & \\
\hline & Total & 474 & 59 & 63 & 4 & \\
\hline
\end{tabular}

${ }^{*} p<0.05,{ }^{* *} p<0.01{ }^{* * *}$ The availability of the information system such as PACS, and dictation system is investigated, as in Table 6 , using the following set of selections: "Live; Live - hospital-wide; Live - departmental; Installation in Process; Service Not Provided; Not Automated; Missing". It is just because such an information system can be applied at the departmental level. On the other hand, the availability of administration or application of EHR functions, such as e-order, clinical documentation, etc. is investigated with the following set of selections: "Yes; No; Not Applicable; Missing" since they are either exist or not. If it is reasonable, the prevalence of some of those functions is separately investigated, as in Tables 9 and 14

Table 9 Prevalence of electronic ordering

\begin{tabular}{|c|c|c|c|c|c|c|c|c|c|}
\hline Questions & Hospital Size & $\begin{array}{l}100 \% \\
\text { (all) }\end{array}$ & $\begin{array}{l}76- \\
100 \%\end{array}$ & $\begin{array}{l}51- \\
75 \%\end{array}$ & $\begin{array}{l}26- \\
50 \%\end{array}$ & $\begin{array}{l}1- \\
25 \%\end{array}$ & $\begin{array}{l}\text { Not } \\
\text { Applicable }\end{array}$ & Missing & $P$ \\
\hline \multirow[t]{5}{*}{$\begin{array}{l}\text { What } \% \text { of all inpatient medication orders } \\
\text { are processed electronically? }\end{array}$} & Large (> = 400 beds) & 0 & $\begin{array}{l}48 \\
(81.35 \%)\end{array}$ & $\begin{array}{l}3 \\
(5.08 \%)\end{array}$ & $\begin{array}{l}1 \\
(1.69 \%)\end{array}$ & $\begin{array}{l}2 \\
(3.38 \%)\end{array}$ & $\begin{array}{l}5 \\
(8.47 \%)\end{array}$ & 41 & 0.856 \\
\hline & $\begin{array}{l}\text { Medium (100-399 } \\
\text { beds) }\end{array}$ & 0 & $\begin{array}{l}123 \\
(81.45 \%)\end{array}$ & $\begin{array}{l}9 \\
(5.96 \%)\end{array}$ & $\begin{array}{l}2 \\
(1.32 \%)\end{array}$ & $\begin{array}{l}4 \\
(2.64 \%)\end{array}$ & $\begin{array}{l}13 \\
(8.6 \%)\end{array}$ & 54 & \\
\hline & Small (6-99 beds) & 0 & $\begin{array}{l}203 \\
(71.22 \%)\end{array}$ & $\begin{array}{l}14 \\
(4.91 \%)\end{array}$ & $\begin{array}{l}9 \\
(3.15 \%)\end{array}$ & $\begin{array}{l}12 \\
(4.21 \%)\end{array}$ & $\begin{array}{l}47 \\
(16.49 \%)\end{array}$ & 10 & \\
\hline & Percentage & $0.0 \%$ & $62.3 \%$ & $4.3 \%$ & $2.0 \%$ & $3.0 \%$ & $10.8 \%$ & $17.5 \%$ & \\
\hline & Total & 0 & 374 & 26 & 12 & 18 & 65 & 105 & \\
\hline \multirow[t]{5}{*}{$\begin{array}{l}\text { What } \% \text { of all inpatient non-medication } \\
\text { orders are processed electronically? }\end{array}$} & Large (> = 400 beds) & 0 & $\begin{array}{l}63 \\
(66.31 \%)\end{array}$ & $\begin{array}{l}5 \\
(5.26 \%)\end{array}$ & $\begin{array}{l}4 \\
(4.21 \%)\end{array}$ & $\begin{array}{l}4 \\
(4.21 \%)\end{array}$ & $\begin{array}{l}19 \\
(20 \%)\end{array}$ & 5 & 0.586 \\
\hline & $\begin{array}{l}\text { Medium (100-399 } \\
\text { beds) }\end{array}$ & 0 & $\begin{array}{l}125 \\
(62.18 \%)\end{array}$ & $\begin{array}{l}17 \\
(8.45 \%)\end{array}$ & $\begin{array}{l}10 \\
(4.97 \%)\end{array}$ & $\begin{array}{l}11 \\
(5.47 \%)\end{array}$ & $\begin{array}{l}38 \\
(18.90 \%)\end{array}$ & 4 & \\
\hline & Small (6-99 beds) & 0 & $\begin{array}{l}180 \\
(61.22 \%)\end{array}$ & $\begin{array}{l}33 \\
(11.22 \%)\end{array}$ & $\begin{array}{l}19 \\
(6.46 \%)\end{array}$ & $\begin{array}{l}20 \\
(6.80 \%)\end{array}$ & $\begin{array}{l}42 \\
(14.28 \%)\end{array}$ & 1 & \\
\hline & Percentage & $0.0 \%$ & $61.3 \%$ & $9.2 \%$ & $5.5 \%$ & $5.8 \%$ & $16.5 \%$ & $1.7 \%$ & \\
\hline & Total & 0 & 368 & 55 & 33 & 35 & 99 & 10 & \\
\hline
\end{tabular}


Table 14 Prevalence of IMS

\begin{tabular}{|c|c|c|c|c|c|c|c|c|c|}
\hline Question & Hospital Size & $\begin{array}{l}100 \% \\
\text { (all) }\end{array}$ & $\begin{array}{l}76- \\
100 \%\end{array}$ & $\begin{array}{l}51- \\
75 \%\end{array}$ & $\begin{array}{l}26- \\
50 \%\end{array}$ & $\begin{array}{l}1- \\
25 \%\end{array}$ & $\begin{array}{l}\text { Not } \\
\text { Applicable }\end{array}$ & Missing & $p$ \\
\hline \multirow[t]{5}{*}{$\begin{array}{l}\text { What } \% \text { of medical images in all other } \\
\text { departments are managed by your IMS? }\end{array}$} & $\begin{array}{l}\text { Large }(>=400 \\
\text { beds) }\end{array}$ & $\begin{array}{l}12 \\
(20.7 \%)\end{array}$ & $\begin{array}{l}19 \\
(32.8 \%)\end{array}$ & $\begin{array}{l}9 \\
(15.5 \%)\end{array}$ & $\begin{array}{l}3 \\
(5.2 \%)\end{array}$ & $\begin{array}{l}7 \\
(12.1 \%)\end{array}$ & $\begin{array}{l}8 \\
(13.8 \%)\end{array}$ & 42 & $p<0.001$ \\
\hline & $\begin{array}{l}\text { Medium (100-399 } \\
\text { beds) }\end{array}$ & $\begin{array}{l}24 \\
(14.8 \%)\end{array}$ & $\begin{array}{l}46 \\
(28.4 \%)\end{array}$ & $\begin{array}{l}20 \\
(12.3 \%)\end{array}$ & $\begin{array}{l}11 \\
(6.8 \%)\end{array}$ & $\begin{array}{l}15 \\
(9.3 \%)\end{array}$ & $\begin{array}{l}46 \\
(28.4 \%)\end{array}$ & 43 & \\
\hline & Small (6-99 beds) & $\begin{array}{l}35 \\
(12.3 \%)\end{array}$ & $\begin{array}{l}40 \\
(14.0 \%)\end{array}$ & $\begin{array}{l}19 \\
(6.7 \%)\end{array}$ & $\begin{array}{l}21 \\
(7.4 \%)\end{array}$ & $\begin{array}{l}23 \\
(8.1 \%)\end{array}$ & $\begin{array}{l}147 \\
(51.6 \%)\end{array}$ & 10 & \\
\hline & Percentage & $11.8 \%$ & $17.5 \%$ & $8.0 \%$ & $5.8 \%$ & $7.5 \%$ & $33.5 \%$ & $15.8 \%$ & \\
\hline & Total & 71 & 105 & 48 & 35 & 45 & 201 & 95 & \\
\hline \multirow[t]{5}{*}{$\begin{array}{l}\text { What } \% \text { of medical images in Radiology } \\
\text { are managed by your IMS? }\end{array}$} & $\begin{array}{l}\text { Large }(>=400 \\
\text { beds) }\end{array}$ & $\begin{array}{l}29 \\
(48.3 \%)\end{array}$ & $\begin{array}{l}26 \\
(43.3 \%)\end{array}$ & $\begin{array}{l}2 \\
(3.3 \%)\end{array}$ & $\begin{array}{l}1 \\
(1.7 \%)\end{array}$ & $\begin{array}{l}0 \\
(0.0 \%)\end{array}$ & $\begin{array}{l}2 \\
(3.3 \%)\end{array}$ & 40 & $p<0.001$ \\
\hline & $\begin{array}{l}\text { Medium (100-399 } \\
\text { beds) }\end{array}$ & $\begin{array}{l}57 \\
(34.8 \%)\end{array}$ & $\begin{array}{l}89 \\
(54.3 \%)\end{array}$ & $\begin{array}{l}11 \\
(6.7 \%)\end{array}$ & $\begin{array}{l}1 \\
(0.6 \%)\end{array}$ & $\begin{array}{l}3 \\
(1.8 \%)\end{array}$ & $\begin{array}{l}3 \\
(1.8 \%)\end{array}$ & 41 & \\
\hline & Small (6-99 beds) & $\begin{array}{l}133 \\
(45.5 \%)\end{array}$ & $\begin{array}{l}71 \\
(24.3 \%)\end{array}$ & $\begin{array}{l}24 \\
(8.2 \%)\end{array}$ & $\begin{array}{l}10 \\
(3.4 \%)\end{array}$ & $\begin{array}{l}11 \\
(3.8 \%)\end{array}$ & $\begin{array}{l}43 \\
(14.7 \%)\end{array}$ & 3 & \\
\hline & Percentage & $36.5 \%$ & $31.0 \%$ & $6.2 \%$ & $2.0 \%$ & $2.3 \%$ & $8.0 \%$ & $14.0 \%$ & \\
\hline & Total & 219 & 186 & 37 & 12 & 14 & 48 & 84 & \\
\hline
\end{tabular}

proportion of hospitals that have adopted such applications and functions in at least half of the hospital varies between 57.3 and 79.3\%. Despite the relationship between hospital size and availability of the applications, there is a significant relationship between the hospital size and the adoption of some EHR applications or functions. For example, the adoption of clinical documents, medication, and non-medication orders is higher among small hospitals compared to larger hospitals. Similarly, the use of CDSS during drug orders is more frequently adopted by small hospitals. Larger hospitals perform better than smaller hospitals only with regard

Table 10 Usage of CDSS

\begin{tabular}{|c|c|c|c|c|c|c|}
\hline Questions & Hospital Size & Yes & No & Not Applicable & Missing & $\mathbf{P}$ \\
\hline \multirow[t]{5}{*}{$\begin{array}{l}\text { Clinical Documentation (Physician / } \\
\text { Nursing Documentation) }\end{array}$} & Large (> = 400 beds) & $\begin{array}{l}63 \\
(65.6 \%)\end{array}$ & $\begin{array}{l}6 \\
(6.3 \%)\end{array}$ & $\begin{array}{l}27 \\
(28.1 \%)\end{array}$ & 4 & $p<0.001$ \\
\hline & Medium (100-399 beds) & $\begin{array}{l}131 \\
(64.5 \%)\end{array}$ & $\begin{array}{l}23 \\
(11.3 \%)\end{array}$ & $\begin{array}{l}49 \\
(24.1 \%)\end{array}$ & 2 & \\
\hline & Small (6-99 beds) & $\begin{array}{l}220 \\
(75.9 \%)\end{array}$ & $\begin{array}{l}37 \\
(12.8 \%)\end{array}$ & $\begin{array}{l}33 \\
(11.4 \%)\end{array}$ & 5 & \\
\hline & Percentage & $69.0 \%$ & $11.0 \%$ & $18.2 \%$ & $1.8 \%$ & \\
\hline & Total & 414 & 66 & 109 & 11 & \\
\hline \multirow[t]{5}{*}{ Medication Orders } & Large (> = 400 beds) & $\begin{array}{l}64 \\
(66.0 \%)\end{array}$ & $\begin{array}{l}4 \\
(4.1 \%)\end{array}$ & $\begin{array}{l}29 \\
(29.9 \%)\end{array}$ & 6 & 0.087 \\
\hline & Medium (100-399 beds) & $\begin{array}{l}144 \\
(71.6 \%)\end{array}$ & $\begin{array}{l}9 \\
(4.5 \%)\end{array}$ & $\begin{array}{l}48 \\
(23.8 \%)\end{array}$ & 4 & \\
\hline & Small (6-99 beds) & $\begin{array}{l}225 \\
(76.5 \%)\end{array}$ & $\begin{array}{l}18 \\
(6.1 \%)\end{array}$ & $\begin{array}{l}51 \\
(17.3 \%)\end{array}$ & 1 & \\
\hline & Percentage & $71.7 \%$ & $5.2 \%$ & $21.3 \%$ & $1.8 \%$ & \\
\hline & Total & 430 & 31 & 128 & 11 & \\
\hline \multirow[t]{5}{*}{ Non-Medication Orders } & Large (> = 400 beds) & $\begin{array}{l}48 \\
(85.7 \%)\end{array}$ & $\begin{array}{l}7 \\
(12.5 \%)\end{array}$ & $\begin{array}{l}1 \\
(1.8 \%)\end{array}$ & 44 & $p<0.001$ \\
\hline & Medium (100-399 beds) & $\begin{array}{l}114 \\
(72.2 \%)\end{array}$ & $\begin{array}{l}43 \\
(27.2 \%)\end{array}$ & $\begin{array}{l}1 \\
(0.6 \%)\end{array}$ & 47 & \\
\hline & Small (6-99 beds) & $\begin{array}{l}182 \\
(65.7 \%)\end{array}$ & $\begin{array}{l}71 \\
(25.6 \%)\end{array}$ & $\begin{array}{l}24 \\
(8.7 \%)\end{array}$ & 18 & \\
\hline & Percentage & $57.3 \%$ & $20.2 \%$ & $4.3 \%$ & $18.2 \%$ & \\
\hline & Total & 344 & 121 & 26 & 109 & \\
\hline
\end{tabular}


Table 11 Usage of CLMA

\begin{tabular}{|c|c|c|c|c|c|c|}
\hline Questions & Hospital Size & Yes & No & Not Applicable & Missing & $\mathbf{P}$ \\
\hline \multirow[t]{5}{*}{$\begin{array}{l}\text { 2nd line of validation for medication prescriptions } \\
\text { which is documented electronically }\end{array}$} & Large ( $>=400$ beds) & $\begin{array}{l}53 \\
(88.3 \%)\end{array}$ & $\begin{array}{l}7 \\
(11.7 \%)\end{array}$ & $\begin{array}{l}0 \\
(0.0 \%)\end{array}$ & 40 & 0.271 \\
\hline & Medium (100-399 beds) & $\begin{array}{l}132 \\
(80.5 \%)\end{array}$ & $\begin{array}{l}32 \\
(19.5 \%)\end{array}$ & $\begin{array}{l}0 \\
(0.0 \%)\end{array}$ & 41 & \\
\hline & Small (6-99 beds) & $\begin{array}{l}230 \\
(79.3 \%)\end{array}$ & $\begin{array}{l}60 \\
(20.7 \%)\end{array}$ & $\begin{array}{l}0 \\
(0.0 \%)\end{array}$ & 5 & \\
\hline & Percentage & $69.2 \%$ & $16.5 \%$ & $0.0 \%$ & $14.3 \%$ & \\
\hline & Total & 415 & 99 & 0 & 86 & \\
\hline \multirow[t]{5}{*}{ Automated Dispensing of medication is available } & Large ( $>=400$ beds) & $\begin{array}{l}3 \\
(5.0 \%)\end{array}$ & $\begin{array}{l}57 \\
(95.0 \%)\end{array}$ & $\begin{array}{l}0 \\
(0.0 \%)\end{array}$ & 40 & $0.022^{*}$ \\
\hline & Medium (100-399 beds) & $\begin{array}{l}1 \\
(0.6 \%)\end{array}$ & $\begin{array}{l}160 \\
(99.4 \%)\end{array}$ & $\begin{array}{l}0 \\
(0.0 \%)\end{array}$ & 44 & \\
\hline & Small (6-99 beds) & $\begin{array}{l}1 \\
(0.3 \%)\end{array}$ & $\begin{array}{l}291 \\
(99.7 \%)\end{array}$ & $\begin{array}{l}0 \\
(0.0 \%)\end{array}$ & 3 & \\
\hline & Percentage & $0.8 \%$ & $84.7 \%$ & $0.0 \%$ & $14.5 \%$ & \\
\hline & Total & 5 & 508 & 0 & 87 & \\
\hline \multirow[t]{5}{*}{$\begin{array}{l}\text { Closed-loop medication administration at the point } \\
\text { of care }\end{array}$} & Large ( $>=400$ beds) & $\begin{array}{l}60 \\
(75.9 \%)\end{array}$ & $\begin{array}{l}19 \\
(24.1 \%)\end{array}$ & $\begin{array}{l}0 \\
(0.0 \%)\end{array}$ & 21 & $p<0.001$ \\
\hline & Medium (100-399 beds) & $\begin{array}{l}117 \\
(62.9)\end{array}$ & $\begin{array}{l}69 \\
(37.1 \%)\end{array}$ & $\begin{array}{l}0 \\
(0.0 \%)\end{array}$ & 19 & \\
\hline & Small (6-99 beds) & $\begin{array}{l}93 \\
(31.8 \%)\end{array}$ & $\begin{array}{l}199 \\
(68.2 \%)\end{array}$ & $\begin{array}{l}0 \\
(0.0 \%)\end{array}$ & 2 & \\
\hline & Percentage & $45.0 \%$ & $47.8 \%$ & $0.0 \%$ & $7.0 \%$ & \\
\hline & Total & 270 & 287 & 0 & 42 & \\
\hline \multirow[t]{5}{*}{$\begin{array}{l}\text { Electronic Medication Administration Record } \\
\text { (EMAR) available at point of care/bedside? }\end{array}$} & Large (> = 400 beds) & $\begin{array}{l}44 \\
(44.0 \%)\end{array}$ & $\begin{array}{l}18 \\
(18.0 \%)\end{array}$ & $\begin{array}{l}38 \\
(38.0 \%)\end{array}$ & 0 & $p<0.001$ \\
\hline & Medium (100-399 beds) & $\begin{array}{l}99 \\
(48.8 \%)\end{array}$ & $\begin{array}{l}65 \\
(32.0 \%)\end{array}$ & $\begin{array}{l}39 \\
(19.2 \%)\end{array}$ & 2 & \\
\hline & Small (6-99 beds) & $\begin{array}{l}105 \\
(36.6 \%)\end{array}$ & $\begin{array}{l}181 \\
(63.1 \%)\end{array}$ & $\begin{array}{l}1 \\
(0.3 \%)\end{array}$ & 7 & \\
\hline & Percentage & $41.3 \%$ & $44.0 \%$ & $13.0 \%$ & $1.5 \%$ & \\
\hline & Total & 248 & 264 & 78 & 9 & \\
\hline
\end{tabular}

${ }^{*} p<0.05$

to second-line verification of medication administration at bedside, which may be explained by available resources, i.e., nurses.

Jha et al. [22], DesRoches et al. [71], and Hikmet et al. [72] showed that small hospitals in the USA are significantly slower to adopt EHR functions than larger hospitals. One reason may be extracted from the study of Thakkar and Davis, which posed that the perception of hospital managers in the USA is that the benefits of EHR adoption are greater in larger hospitals than in smaller ones [73]. In addition, large hospitals in the USA may have larger financial and human resources to dedicate to the adoption of EHR functions than smaller hospitals might have. Turkey, as a centralized state, may be smaller than the USA, but the $\mathrm{MoH}$ can actively regulate all state hospitals nation-wide. Small hospitals in Turkey may use their size as an advantage to change more quickly than large hospitals. Nevertheless, when we consider the second line validation for medication administration at bedside, larger hospitals in Turkey also perform better, most probably due to more available financial and human resources, as suggested by Thakkar and Davis [73].

When we consider the concepts of basic and comprehensive EHR functions as defined by Jha et al. [21], by comparing them with EMRAM stages (as expressed in Table 1), we found that $63 \%$ of all hospitals surveyed in Turkey have at least basic EHR functions, and 36\% have comprehensive EHR functions. This result is better than the results of Korean hospitals in 2017 [29], but lower than that of hospitals in the USA in 2015 [24] and 2017 [25], as depicted in Table 15.

Additionally, the Turkish experience summarized in this study strengthens the claim [34-36] that following a bottom-up approach to encouraging the adoption of EHRs in public hospitals employed in the USA, is more successful than the top-down approach used in the UK. 
Table 12 Usage of The Five Rights of Medication Administration at bedside

\begin{tabular}{|c|c|c|c|c|c|c|}
\hline Questions & Hospital Size & Auto-identified & Yes & No & Missing & $\mathbf{P}$ \\
\hline \multirow[t]{15}{*}{$\begin{array}{l}\text { Which of the following is auto-identified } \\
\text { during bedside medication administration? }\end{array}$} & \multirow[t]{5}{*}{ Large ( $>=400$ beds) } & Medication & $\begin{array}{l}58 \\
(69.0 \%)\end{array}$ & $\begin{array}{l}26 \\
(31.0 \%)\end{array}$ & 16 & \multirow[t]{5}{*}{$0.028^{*}$} \\
\hline & & Nurse & $\begin{array}{l}43 \\
(51.2 \%)\end{array}$ & $\begin{array}{l}41 \\
(48.8 \%)\end{array}$ & 16 & \\
\hline & & Patient & $\begin{array}{l}57 \\
(67.9 \%)\end{array}$ & $\begin{array}{l}27 \\
(31.1 \%)\end{array}$ & 16 & \\
\hline & & Percentage & $52.7 \%$ & $31.3 \%$ & $16.0 \%$ & \\
\hline & & Total & 158 & 94 & 48 & \\
\hline & \multirow[t]{5}{*}{ Medium (100-399 beds) } & Medication & $\begin{array}{l}124 \\
(61.7 \%)\end{array}$ & $\begin{array}{l}77 \\
(38.3 \%)\end{array}$ & 4 & \multirow[t]{5}{*}{$0.003^{* *}$} \\
\hline & & Nurse & $\begin{array}{l}95 \\
(47.3 \%)\end{array}$ & $\begin{array}{l}106 \\
(52.7 \%)\end{array}$ & 4 & \\
\hline & & Patient & $\begin{array}{l}125 \\
(62.2 \%)\end{array}$ & $\begin{array}{l}76 \\
(37.8 \%)\end{array}$ & 4 & \\
\hline & & Percentage & $55.9 \%$ & $42.1 \%$ & $2.0 \%$ & \\
\hline & & Total & 344 & 259 & 12 & \\
\hline & \multirow[t]{5}{*}{ Small (6-99 beds) } & Medication & $\begin{array}{l}104 \\
(35.5 \%)\end{array}$ & $\begin{array}{l}189 \\
(64.5 \%)\end{array}$ & 2 & \multirow[t]{5}{*}{0.422} \\
\hline & & Nurse & $\begin{array}{l}99 \\
(33.8 \%)\end{array}$ & $\begin{array}{l}194 \\
(66.2 \%)\end{array}$ & 2 & \\
\hline & & Patient & $\begin{array}{l}114 \\
(38.9 \%)\end{array}$ & $\begin{array}{l}179 \\
(61.1 \%)\end{array}$ & 2 & \\
\hline & & Percentage & $35.8 \%$ & $63.5 \%$ & $0.7 \%$ & \\
\hline & & Total & 317 & 562 & 6 & \\
\hline
\end{tabular}

${ }^{*} p<0.05,{ }^{* *} p<0.01$

Measuring country-wide EHR adoption is becoming widespread in the literature. Not only developed and developing countries, low- and middle-income countries such as Kenya [74] and Ghana [75] are also measuring their EHR adoption level. There are several models used in those studies. The survey used by Jha et al. in 2009 [21] is the pioneer of many studies; the same survey has been used by other researchers like in Korea [29]. On the other hand, many countries, such as Japan [33], China [30], developed their maturity models. Finally, some countries such as Canada [55], Portugal [54], and Turkey, as in this study, preferred to use HIMSS EMRA $\mathrm{M}$ as a maturity model.
Besides country-wide studies, there is an increasing number of studies about EHR adoption in the health system. The most recent review published in 2019 criticized 18 studies between 2005 and 2017, which are applied in different healthcare facilities from primary to tertiary healthcare services [76].

\section{Conclusion}

Measuring the national adoption rates of EHR functions provides critical information and insights for healthcare policymakers. Despite all practical difficulties, studies measuring the overall adoption level of EHRs are increasing in number. This study is the first one to cover

Table 13 IMS Integration with EMR

\begin{tabular}{|c|c|c|c|c|c|c|}
\hline Question & Hospital Size & Yes & No & Not Applicable & Missing & $\mathbf{P}$ \\
\hline \multirow[t]{5}{*}{$\begin{array}{l}\text { Is your IMS solution integrated with your } \\
\text { Electronic Medical Record (EMR)? }\end{array}$} & Large (> = 400 beds) & $\begin{array}{l}57 \\
(95.0 \%)\end{array}$ & $\begin{array}{l}1 \\
(1.7 \%)\end{array}$ & $\begin{array}{l}2 \\
(3.3 \%)\end{array}$ & 40 & $p<0.001$ \\
\hline & Medium (100-399 beds) & $\begin{array}{l}155 \\
(95.7 \%)\end{array}$ & $\begin{array}{l}4 \\
(2.5 \%)\end{array}$ & $\begin{array}{l}3 \\
(1.9 \%)\end{array}$ & 43 & \\
\hline & Small (6-99 beds) & $\begin{array}{l}236 \\
(81.7 \%)\end{array}$ & $\begin{array}{l}10 \\
(3.5 \%)\end{array}$ & $\begin{array}{l}43 \\
(14.9 \%)\end{array}$ & 6 & \\
\hline & Percentage & $74.7 \%$ & $2.5 \%$ & $8.0 \%$ & $14.8 \%$ & \\
\hline & Total & 448 & 15 & 48 & 89 & \\
\hline
\end{tabular}


Table 15 Comparison EHR adoption of the USA (in 2017), Korea (in 2017) and Turkey (in 2014-17)

\begin{tabular}{llll}
\hline Hospital Size & USA & Korea & Turkey \\
\hline Basic EHR functions & $41.4 \%$ & NA & $27.1 \%$ \\
Comprehensive EHR functions & $39.1 \%$ & NA & $36 \%$ \\
Hospitals having at least basic EHR functions & $80.5 \%$ & $58.1 \%$ & $63.1 \%$ \\
\hline
\end{tabular}

all state hospitals in a country using the EMRAM model developed by HIMSS. As the first nation-wide study in Turkey, the results may enable researchers to compare among countries like the USA, Korea, and China. Contrary to the conclusions of previous studies [22, 71, 72], this study found that smaller hospitals are better at adopting most EHR functions, with the exception of second-line validation for medication administration at bedside.

Additionally, as in the USA [34-36], this study found that after all required standardization and infrastructural studies, applying a bottom-up approach to adopting EHR functions in state hospitals has been successful in Turkey.

The results of this study are used by the $\mathrm{MoH}$ of Turkey to disseminate the benefits of EHR functions across the country. In consideration of studies showing the effect of using EHR functions on increasing healthcare quality, the Turkish MoH's experience of using EMRAM may suggest that measuring adoption rates of EHR functions can be a good starting point for a healthcare authority to set targets to improve healthcare quality.

For further studies, it may be interesting to focus on measuring the correlation of EHR adoption level and healthcare quality scores measured by international standards, such as Joint Commission International, etc.

\footnotetext{
Abbreviations

AMD: Automated Medication Dispensing; CDR: Clinical Data Repository; CDSS: Clinical Decision Support Systems; CPOE: Computerized Physician Order Entries; EHR: Electronic Health Record; eMAR: Electronic Medication Administration Records; EMB: Electronic Medical Billing; EMRAM: Electronic Medical Record Maturity Model; EO: Electronic Ordering; HIE: Health Information Exchange; HIMSS: Healthcare Information and Management Systems Society; HIS: Hospital Information System; HL7: Health Level Seven International; ICD-10: International Classification of Diseases, 10th Revision; IMS: Image Management Systems; IT: Information Technology; MEG: Model of EHR Grading; MHLW: Ministry of Health, Labour and Welfare;

MoH: Ministry of Health; MS: Medication Safety; NHDD: National Health Data Dictionary; PACS: Picture Archiving and Communication Systems; PAS: Patient Administration Systems; PD: Physician Documents; RFID: RadioFrequency Identification; SA: Software Applications; SKS: Turkish national healthcare quality standards; SU: Software Usage; UK: United Kingdom; US: United States; USA: United States of America
}

\section{Acknowledgments}

The authors thank Dr. Korhan An for his valuable guidance for survey studies, and to Pakize Yigit for performing statistical analyses. The authors also thank the collaboration groups, the HIMSS Analytics Team, and the MoH Team, for their valuable efforts for encouraging hospitals to participate in the survey studies and workshops and for their contributions to the research.
HIMSS Analytics Team: Suna Kirdag Mahir, Kubra Aycil, Beytiye O. Elmas, Esra Volkan, Zeynep Altinbas, and Gizem Gencyurek.

MoH Team: Esra Zehir, Berrin Gundogdu, Mert Ozcan, Ceyhan Vardar, Behcet Altinli, and Jale Sungur Hasancebi.

\section{Authors' contributions}

IK generated the survey database and made relevant analyses, conducted the literature review, and was a major contributor in writing the manuscript. JR helped in designing the survey and the quality control mechanism, and contributed to writing the manuscript. SG enhanced the literature review and constructed the tables in the manuscript. SB, MMU, and IY planned the regional workshops for state hospitals, trained the hospital delegates, contributed to gathering the surveys from hospitals, and reviewed the manuscript. The author(s) read and approved the final manuscript.

\section{Funding}

Not applicable.

\section{Availability of data and materials}

The datasets generated and analyzed during the current study are not publicly available due to $\mathrm{MoH}$ regulations but are available from the corresponding author upon reasonable request.

Ethics approval and consent to participate

Not applicable.

\section{Consent for publication}

Not applicable.

\section{Competing interests}

The authors declare that they have no competing interests.

\section{Author details}

${ }^{1}$ Department of Health System Engineering, Istanbul Medipol University, 34810 Istanbul, Turkey. ${ }^{2}$ HIMSS Analytics for Europe and Latin America, Huddersfield, UK. ${ }^{3}$ Ministry of Health, 06800 Ankara, Turkey. ${ }^{4}$ Istanbul Medipol University, 34810 Istanbul, Turkey.

Received: 16 November 2019 Accepted: 27 September 2020 Published online: 21 October 2020

\section{References}

1. Turkish MoH. EHR Definition [Internet]. 2020 [cited 2020 Jun 27]. Available from: https://dijitalhastane.saglik.gov.tr/TR,4874/ehr-electronic-healthrecord\%2D\%2D-esk-elektronik-saglik-kaydi.html.

2. Latha NA, Rama Murthy B, Sunitha U. Electronic health record [Internet]. Int J Engineering Res Technology (IJERT). 2012; Available from: www.jert.org.

3. Fernando B, Kalra D, Morrison Z, Byrne E, Sheikh A. Benefits and risks of structuring and/or coding the presenting patient history in the electronic health record : systematic review. BMJ Qual Saf. 2012;21:337-46.

4. Koppel R, Cohen A, Abaluck B, Localio AR, Kimmel SE, Strom BL. Role of computerized Physician order entry Systems in Facilitating Medication Errors Ross. Am Med Assoc. 2005;293(10):1197-203.

5. Wolfstadt JI, Gurwitz JH, Field TS, Lee M, Kalkar S, Wu W, et al. The Effect of Computerized Physician Order Entry with Clinical Decision Support on the Rates of Adverse Drug Events: A Systematic Review. J Gen Intern Med [Internet]. 2008;23(4):451-8 [cited 2018 Aug 6] Available from: http://link. springer.com/10.1007/s11606-008-0504-5.

6. Kaushal R, Shojania KG, Bates DW. Effects of computerized Physician order entry and clinical decision support systems on medication safety. Arch Intern Med. 2003;163(12):1409.

7. Poon EG, Blumenthal D, Jaggi T, Honour MM, Bates DW, Kaushal R. Overcoming Barriers To Adopting And Implementing Computerized Physician Order Entry Systems In U.S. Hospitals. Health Aff [Internet]. 2004; 23(4):184-90 [cited 2018 Aug 6] Available from: http://www.healthaffairs. org/doi/10.1377/hlthaff.23.4.184.

8. Henderson D, Lunak R, Markiewicz E, Tobin CC. Closed loop medication use system and method. 2003 Jun 19 [cited 2018 Aug 8];2(12). Available from: https://patents.google.com/patent/US8478604B2/en.

9. Franklin BD, O'Grady K, Donyai $P$, Jacklin A, Barber N. The impact of a closed-loop electronic prescribing and administration system on prescribing 
errors, administration errors and staff time: a before-and-after study. Qual Saf Heal Care. 2007;16(4):279-84.

10. Lester D. Provider risk factors for medication administration error alerts: analyses of a large-scale closed-loop medication administration system using RFID and barcode. Clin Neuropsychiatry. 2009;6(5):188-91.

11. Panosh N, Rew R, Sharpe M. Effect of closed-loop order processing on the time to initial antimicrobial therapy. Am J Heal Pharm. 2012;69(16):1423-6.

12. Medanet. Closed Loop Medication Administration: Electronic Verification of "the Five Rights" - Medanets [Internet]. 2017 [cited 2018 Aug 8]. Available from: https://medanets.com/en/blog/closed-loop-medicationadministration-electronic-verification-of-the-five-rights/.

13. Bassa A, del Val M, Cobos A, Torremade E, Bergonon S, Crespo C, et al. Impact of a clinical decision support system on the Management of Patients with hypercholesterolemia in the primary healthcare setting. Dis Manag Heal Outcomes [Internet]. 2005;13(1):65-72 Available from: http:// link.springer.com/10.2165/00115677-200513010-00007.

14. Berner ES. Clinical Decision Support Systems Theory and Practice [Internet]. Second Edi. Berner ES, editor. New York, NY: Health Informatics Series; 2007 [cited 2018 Aug 6]. (Health Informatics). Available from: http://link.springer. com/10.1007/978-0-387-38319-4.

15. Beeler PE, Bates DW, Hug BL. Clinical Decision Support Systems. Swiss Med Wkly [Internet]. 2014 Dec 23 [cited 2018 Aug 6]; Available from: http://doi. emh.ch/smw.2014.14073.

16. Choplin RH, Boebme li JM, MCD M. Picture Archiving and Communication Systems: An Overview. Radio Graph [Internet]. 1992;12:127-9 [cited 2018 Jul 3] Available from: https://pubs.rsna.org/doi/pdf/10.1148/radiographics.12.1.1 734458.

17. Connection, Connection we solve IT. Electronic Medication Administration (eMAR) [Internet]. Connection we solve IT. 2018 [cited 2018 Aug 9]. Available from: https://www.connection.com/IPA/PM/Solutions/ HealthConnection/Solutions/POC/Medication.

18. Kılıç T. Digital hospital: An example of best practice. Int J Heal Sci Res Policy [Internet]. 2016;1(2). Available from: http://dergipark.gov.tr/download/articlefile/329474.

19. Ayres D, Soar J, Conrick M. Health information systems. In: Conrick M, editor. Health Informatics: Transforming Healthcare with Technology; 2006.

20. Rodriguez-Martinez M, Valdivia H, Rivera J, Seguel J, Greer M. MedBook: A cloud-based healthcare billing and record management system. In: 2012 IEEE 5th International Conference on Cloud Computing, CLOUD 2012. Honolulu; 2012. p. 899-905.

21. Jha AK, DesRoches CM, Campbell EG, Donelan K, Rao RS, Ferris GT, et al. Use of electronic health records in US hospitals. N Engl J Med. 2009;360:1628-38.

22. Jha AK, Burke MF, DesRoches C, Joshi MS, Kralovec P, Campbell EG, et al. Progress toward meaningful use: hospitals' adoption of electronic health records. Am J Manag Care [Internet]. 2011 Dec [cited 2019 Jun 15];17(12): 117-24. Available from: http://www.ncbi.n/m.nih.gov/pubmed/22216770.

23. Adler-Milstein J, M. DesRoches C, Furukawa MF, Worzala C, Charles D, Kralovec $P$, et al. more than half of US hospitals have at least a basic EHR, but stage 2 criteria remain challenging for most. Health Aff [Internet] 2014; 33(9):1664-71. Available from: http://content.healthaffairs.org/content/33/ 9/1664.full.

24. Adler-Milstein J, Desroches CM, Kralovec P, Foster G, Worzala C, Charles D, et al. Electronic Health Record Adoption In US Hospitals: Progress Continues, But Challenges Persist. Health Aff [Internet]. 2015;34(12):2174-80 [cited 2019 Jun 15] Available from: https://www.healthaffairs.org/doi/pdf/1 0.1377/hlthaff.2015.0992.

25. Adler-Milstein J, Holmgren AJ, Kralovec P, Worzala C, Searcy T, Patel V. Electronic health record adoption in US hospitals: the emergence of a digital "advanced use" divide. J Am Med Informatics Assoc [Internet]. 2017 Nov 1 [cited 2019 Sep 2];24(6):1142-8. Available from: http://academic.oup. com/jamia/article/24/6/1142/4091350/Electronic-health-record-adoption-inUS-hospitals.

26. Woong Park R, Shin S, Ouk Ahn J, Hwang SC. Computerized Physician Order Entry and Electronic Medical Record Systems in Korean Teaching and General Hospitals: Results of a 2004 Survey. J Am Med Informatics Assoc [Internet]. 2005;12(6):642-7 [cited 2019 Jun 15] Available from: www.hira.or.kr.

27. Ash JS, Gorman PN, Hersh WR. Physician order entry in U.S. hospitals. Proceedings AMIA Symp [Internet]. 1998 [cited 2019 Sep 2];235-9. Available from: http://www.ncbi.nlm.nih.gov/pubmed/9929217.

28. Yoon D, Chang B-C, Kang SW, Bae H, Park RW. Adoption of electronic health records in Korean tertiary teaching and general hospitals. Int J Med Inform
[Internet]. 2012;81(3):196-203 [cited 2019 May 29] Available from: https:// www.sciencedirect.com/science/article/pii/S1386505611002437.

29. Kim Y-G, Jung K, Park Y-T, Shin D, Yeon Cho S, Yoon D, et al. Rate of electronic health record adoption in South Korea: A nation-wide survey. Int J Med Inform [Internet]. 2017 May 1 [cited 2019 May 29];101:100-7. Available from: https://0210063c2-y-https-www-sciencedirect-com.proxy. medipol.deep-knowledge.net/science/article/pii/S1386505617300400.

30. Shu T, Liu H, Goss FR, Yang W, Zhou L. EHR adoption across China's tertiary hospitals: A cross-sectional observational study. Int J Med Inform [Internet]. 2014 Feb 1 [cited 2019 May 27];83(2):113-21. Available from: https://021 0062fx-y-https-www-sciencedirect-com.proxy.medipol.deep-knowledge.net/ science/article/pii/S1386505613001871.

31. Emmanouilidou M, Burke M. A thematic review and a policy-analysis agenda of Electronic Health Records in the Greek National Health System. Health Policy (New York) [Internet]. 2013 [cited 2019 May 29];109:31-7. Available from: https://doi.org/10.1016/j.healthpol.2012.09.010.

32. Alsulame K, Khalifa M, Househ M. E-Health status in Saudi Arabia: A review of current literature. 2016 [cited 2019 May 29]; Available from: https://pdf. sciencedirectassets.com/280958/1-s2.0-S2211883716X00032/1-s2.0-S2211883 716000137/main.pdf?x-amz-security-token=AgoJb3J.jz2luX2 VjEEYaCXVZLWhc3QtMSJHMEUCIDw\%2BwWDPTA4TIBx5tWdqYNmvgU6 MTmcNgm7kQfQWrYNjAiEA1pWHG4n0mLSe5gNkhGes3jc6aT61\%2 BMdfvWpmeWw5.

33. Kanakubo T, Kharrazi H. Comparing the Trends of Electronic Health Record Adoption Among Hospitals of the United States and Japan. J Med Syst [Internet]. 2019 Jul 1 [cited 2020 Jun 28];43(7):1-13. Available from: https:// link.springer.com/article/10.1007/s10916-019-1361-y.

34. Sheikh A, Jha A, Cresswell K, Greaves F, Bates DW. Adoption of electronic health records in UK hospitals: lessons from the USA. Lancet [Internet]. 2014 [cited 2020 Feb 23];384:8-9. Available from: https://doi.org/10.1016/S01406736

35. Johnson OA, F Fraser HS, Wyatt JC, Walley JD. Electronic health records in the UK and USA. Lancet [Internet]. 2014 [cited 2020 Feb 23];384:954. Available from: http://thevitalityinstitute.org/.

36. Wilson K, Khansa L. Migrating to electronic health record systems: a comparative study between the United States and the United Kingdom. Health Policy (New York). 2018;122(11):1232-9.

37. Atun R, Aydın S, Chakraborty S, Sümer S, Aran M, Gürol I, et al. Universal health coverage in Turkey: enhancement of equity. Lancet [Internet]. 2013; 382(9886):65-99 [cited 2019 Jan 5] Available from: https://www. sciencedirect.com/science/article/pii/S014067361361051X.

38. Kose I, Akpinar N, Gurel M, Arslan Y, Ozer H, Yurt N, et al. Turkey's National Health Information System (NHIS). In: e-Challenges. Stockholm; 2008. p. 170-177.

39. $\mathrm{MoH}$. Health Coding Reference Server (HCRS) [Internet]. Ministry of Health. 2009 [cited 2016 Nov 26]. Available from: https://skrs3.sagliknet.saglik.gov.tr.

40. Demir M. Kodlama Kültürsüzlüğü Örnek 1: ICD-10. SD Platform [Internet]. 2007 Mar [cited 2018 Aug 23];36-9. Available from: http://www.sdplatform. com/Dergi/25/Kodlama-Kultursuzlugu-Ornek-1-ICD-10.aspx.

41. Namli T, Aluc G, Sinaci AA, Kose I, Akpinar N, Gurel M, et al. Testing the Conformance and Interoperability of NHIS to Turkey 's HL7 Profile. In: 9th International HL7 Interoperability Conference [Internet]. Crete, Greece; 2008. p. 63-8. Available from: http://www.srdc.metu.edu.tr/webpage/ publications/2008/11.pdf.

42. Kabak Y, Dogac A, Kose I, Akpinar N, Gurel M, Arslan Y, et al. The Use of HL7 CDA in the National Health Information System ( NHIS ) of Turkey. In: 9th International HL7 Interoperability Conference. Crete, Greece; 2008. p. 49-55.

43. SGK. Sağlık Uygulama Tebliği (SUT). 2005.

44. Kose I. Family Physician Information System (Aile Hekimliği Bilgi Sistemi). SD Platform [Internet]. 2010 Jun [cited 2018 Aug 23];22-5. Available from: http://www.sdplatform.com/Yazilar/Konuk-Yazilar/445/Aile-Hekimligi-BilgiSistemi.aspx.

45. Kose I, Arslan Y. Patients will enable booking from public hospitals (Vatandaş hastaneye randevu ile gidecek). SD Platform [Internet]. 2007 Dec [cited 2018 Aug 23];46-9. Available from: http://www.sdplatform.com/ Yazilar/Konuk-Yazilar/465/Natandas-hastaneye-randevu-ile-gidecek.aspx.

46. Kose I. SGK will be the unique actor in healthcare by means of health information systems (Sağlık bilişimi ile SGK sağlıkta tek aktör olmaya doğru ilerliyor). SD Platform [Internet]. 2008 Mar [cited 2018 Aug 23];66-9. Available from: http://www.sdplatform.com/Yazilar/Konuk-Yazilar/466/Saglikbilisimi-ile-SGK-saglikta-tek-aktor-olmaya-dogru-ilerliyor.aspx. 
47. MoH. Telemedicine System of Turkey [Internet]. 2020 [cited 2020 Feb 16]. Available from: https:/teletip.saglik.gov.tr/\#banner.

48. Kose I. Health information systems in Turkey (Sağlık Bilgi Sistemleri: SağlıkNet, e-Nabız, vb.). In: Özen O, editor. Sağlık Diplomasisi. 1st ed. İstanbul; 2017. p. 221-252.

49. Birinci Ș. Kişisel sağlık kaydı ve Türkiye örneği: e-Nabız. SD Platform [Internet]. 2018 Mar [cited 2018 Aug 23];24-7. Available from: http://www. sdplatform.com/Dergi/1088/Kisisel-saglik-kaydi-ve-Turkiye-ornegi-e-Nabiz. aspx.

50. Ministry of Health T. The percentage of e-prescription on September of 2018 [Internet]. 2018 [cited 2020 Feb 10]. Available from: https:// khgmistatistikdb.saglik.gov.tr/TR,50461/eylul-2018\%2D\%2Dillere-gore-erecete-oranlari.html.

51. T.C. Sağlık Bakanlığı. Strateji Geliştirme Başkanlığı [Internet]. 2013 [cited 2018 Jul 4]. Available from: https://sgb.saglik.gov.tr/.

52. HIMSS. About [Internet]. [cited 2019 Jan 2]. Available from: https://www, himss.org/about-himss.

53. HIMSS. Stage 6 \& 7 Achievement | HIMSS Analytics - North America [Internet]. HIMSS Analytics. 2020 [cited 2020 Jun 28]. Available from: https:// www.himssanalytics.org/stage-6-7-achievement.

54. Pereira S, Silva L, Machado J, Cabral A. The Clinical Informatization in Portugal An Approach to the National Health Service Certification. Int J Reliab Qual E-Healthcare [Internet]. 2020 Apr [cited 2020 Jun 28];9(2):34. Available from: www.igi-global.com/chapter/strategic-approach-healthinteroperability-

55. Chong J, Jason T, Jones M, Larsen D. A model to measure self-assessed proficiency in electronic medical records: validation using maturity survey data from Canadian community-based physicians. Int J Med Inform. 2020 Jun 1;141:104218.

56. Cresswell K, Sheikh A, Krasuska M, Heeney C, Dean Franklin B, Lane W, et al. Reconceptualising the digital maturity of health systems. Lancet Glob Heal [Internet]. 2019 [cited 2020 Jun 28];1:e200-1. Available from: https://www.

57. HIMSS Analytics. Electronic Medical Record Adoption Model | HIMSS Analytics - North America [Internet]. 2017 [cited 2018 Jul 26]. Available from: https://www.himssanalytics.org/emram.

58. HIMSS. Continuity of Care Maturity Model (CCMM) [Internet]. HIMSS Analytics. 2018 [cited 2019 Jan 5]. Available from: https://www. himssanalytics.org/CCMM.

59. HIMSS. Adoption Model for Analytics Maturity [Internet]. HIMSS Analytics. 2018 [cited 2019 Jan 5]. Available from: https://www.himssanalytics.org/ AMAM.

60. HIMSS. Infrastructure Adoption Model (INFRAM) [Internet]. HIMSS Analytics. 2018 [cited 2019 Jan 5]. Available from: https://www.himssanalytics.org/ infram.

61. Furukawa M, Pollack E. Achieving HIMSS Stage 7 designation for EMR adoption. Nurs Manag [Internet]. 2020 Jan 1 [cited 2020 Jun 28];51(1):10-2. Available from: http://journals.lww.com/10.1097/01.NUMA.0000617044.5 7943.e1.

62. Bora Başara B, Aygün A, Çağlar Soytutan I. Sağlık İstatistikleri Yıllığı 2017 Haber Bülteni [Internet]. Ankara; 2018 [cited 2019 Jan 27]. Available from: https://dosyamerkez.saglik.gov.tr/Eklenti/27344,saglik-istatistikleri-yilligi-2017haber-bultenipdf.pdf?0.

63. Linder JA, Ma J, Bates DW, Middleton B, Stafford RS. Electronic Health Record Use and the Quality of Ambulatory Care in the United States. Arch Intern Med [Internet]. 2007 Jul 9 [cited 2017 May 8];167(13):1400-5. Available from: http://archinte.jamanetwork.com/article.aspx?doi=10.1001/ archinte.167.13.1400

64. Crosson JC, Ohman PA, Cohen DJ, Clark EC, Crabtree BF. Typical Electronic Health Record Use in Primary Care Practices and the Quality of Diabetes Care. Ann Fam Med [Internet]. 2012 [cited 2018 Aug 7];10(3):221-7. Available from: http://www.annfammed.org/content/10/3/221.full.pdf.

65. Raglan GB, Margolis B, Paulus RA, Schulkin J. Electronic Health Record Adoption among Obstetrician/Gynecologists in the United States. J Healthc Qual [Internet]. 2015;00(0):1. Available from: http://content.wkhealth.com/ linkback/openurl?sid=WKPTLP:landingpage\&an=01445442-900000000-99934.

66. Adler-Milstein J, Everson J, Lee S-YD. EHR Adoption and Hospital Performance: Time-Related Effects. Health Serv Res [Internet]. 2015 Dec 1 [cited 2018 Feb 19];50(6):1751-71. Available from: http://doi.wiley.com/1 0.1111/1475-6773.12406.

67. Wise LC, Bostrom J, Crosier JA, White S, Caldwell R. Cost-benefit analysis of an automated medication system. Nurs Econ [Internet]. 1996 [cited 2020
Feb 16];224+. Available from: https://go.gale.com/ps/anonymous?id=GALE\% 7CA19064677\&sid=googleScholar\&v=2.1\&it=r\&linkaccess=abs\&issn=0746173 $9 \& p=A O N E \& s w=W$.

68. Dobson G, Sullivan S, Tilson V, Webster D. Reducing costs of managing medication inventory in automated dispensing system in hospital units. SSRN Electron J. 2018

69. Dulong D, Wehba S, Comer D, Stark J, Kurtz M, Trohimovich B. Method and apparatus for providing medication administration warnings [Internet]. US20010056358A1, 2001 [cited 2019 May 18]. Available from: https:// patents.google.com/patent/US20010056358A1/en.

70. Williams D. Medication errors. J R Coll Physicians Edinbg [Internet]. 2007 [cited 2019 May 18];(37):343-7. Available from: http://www.rcpe.ac.uk/sites/ default/files/williams_1.pdf.

71. DesRoches CM, Worzala C, Joshi MS, Kralovec P, Jha AK. Small, Nonteaching, And Rural Hospitals Continue To Be Slow In Adopting Electronic Health Record Systems. Health Aff [Internet]. 2012 May 2 [cited 2019 Sep 7];31(5): 1092-9. Available from: http://www.healthaffairs.org/doi/10.1377/hlthaff.2 012.0153 .

72. Hikmet N, Bhattacherjee A, Menachemi N, Kayhan VO, Brooks RG. The role of organizational factors in the adoption of healthcare information technology in Florida hospitals. Health Care Manag Sci. 2008;11:1-9.

73. Thakkar M, Davis DC. Risks, barriers, and benefits of EHR systems: a comparative study based on size of hospital. Perspect Heal Inf Manag [Internet]. 2006;3(5):1-19 [cited 2019 Sep 7] Available from: http://www.ncbi. nlm.nih.gov/pubmed/18066363.

74. Muinga N, Magare S, Monda J, English M, Fraser H, Powell J, et al. Survey of Electronic Health Record (EHR) Systems in Kenyan Public Hospitals: A mixed-methods survey (Preprint). JMIR Med Informatics [Internet]. 2020 [cited 2020 Jun 28];1-22. Available from: https://preprints.jmir.org/ preprint/12995.

75. Abdulai A-F, Adam F. Health providers' readiness for electronic health records adoption: A cross-sectional study of two hospitals in northern Ghana. Torpey K, editor. PLoS One [Internet]. 2020 Jun 4 [cited 2020 Jun 28]; 15(6):e0231569. Available from: https://dx.plos.org/10.1371/journal.pone. 0231569.

76. Sadoughi F, Khodaveisi T, Ahmadi H. The used theories for the adoption of electronic health record: a systematic literature review. Health Technol (Berl) [Internet]. 2019 [cited 2020 Jun 28];9:383-400. Available from: https://doi. org/10.1007/s12553-018-0277-8.

\section{Publisher's Note}

Springer Nature remains neutral with regard to jurisdictional claims in published maps and institutional affiliations.

Ready to submit your research? Choose BMC and benefit from:

- fast, convenient online submission

- thorough peer review by experienced researchers in your field

- rapid publication on acceptance

- support for research data, including large and complex data types

- gold Open Access which fosters wider collaboration and increased citations

- maximum visibility for your research: over $100 \mathrm{M}$ website views per year

At BMC, research is always in progress.

Learn more biomedcentral.com/submissions 